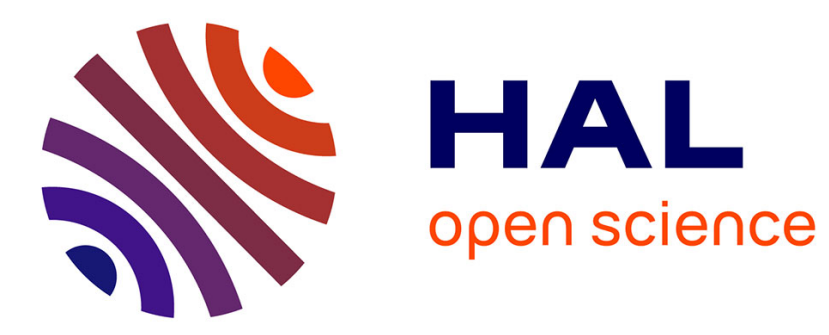

\title{
Airflow characteristics and thermal comfort generated by a multi-cone ceiling diffuser with and without inserted lobes
}

\author{
P. Bragança, K. Sodjavi, Amina Meslem, Laurent Serres
}

\section{- To cite this version:}

P. Bragança, K. Sodjavi, Amina Meslem, Laurent Serres. Airflow characteristics and thermal comfort generated by a multi-cone ceiling diffuser with and without inserted lobes. Building and Environment, 2016, 108, pp.143-158. 10.1016/j.buildenv.2016.08.029 . hal-01371986

HAL Id: hal-01371986

https://hal-univ-rennes1.archives-ouvertes.fr/hal-01371986

Submitted on 9 Nov 2016

HAL is a multi-disciplinary open access archive for the deposit and dissemination of scientific research documents, whether they are published or not. The documents may come from teaching and research institutions in France or abroad, or from public or private research centers.
L'archive ouverte pluridisciplinaire HAL, est destinée au dépôt et à la diffusion de documents scientifiques de niveau recherche, publiés ou non, émanant des établissements d'enseignement et de recherche français ou étrangers, des laboratoires publics ou privés. 


\section{AIRFLOW CHARACTERISTICS AND THERMAL COMFORT GENERATED BY A MULTI-CONE CEILING DIFFUSER WITH AND WITHOUT INSERTED LOBES}

Pierre Bragança ${ }^{1}$, Kodjovi Sodjavi ${ }^{1}$, Amina Meslem ${ }^{2, *}$, Laurent Serres ${ }^{2}$

${ }^{1}$ LaSIE, University of La Rochelle, Pôle Sciences et Technologie, avenue Michel Crépeau, 17042, La Rochelle, France

${ }^{2}$ LGCGM EA3913, University of Rennes 1, 3 rue du Clos Courtel, BP 90422, 35704 Rennes Cedex 7, France, *Corresponding author: amina.meslem@univ-rennes1.fr

\section{ABSTRACT}

Mixing ventilation is commonly used to control thermal comfort in a room by means of air jets. In summer conditions, the occupants often complain of thermal discomfort and draft problems, because the cooling airflow penetrates the occupied zone under buoyancy force effect, with high speeds and low temperatures. To reduce these problems, the jet trajectory could be maintained away from the occupant, by means of a radial jet attached to the ceiling. One great limitation of most radial ceiling diffusers is their inability to correctly mix the jet to the ambient air, and the use of the Coanda effect may worsen this issue because it reduces jet induction. The design of the diffuser itself to enhance the jet mixing with the ambient air by means of inserted lobes was recently proposed in an European patent. In this paper, an experimental investigation is made on airflow pattern and thermal comfort generated in a full scale model room by a ceiling diffuser, and its performance is compared with the same diffuser equipped with inserted lobes. The jet flow from the diffuser and its interaction with a heated manikin simulating an occupant were analyzed with the whole-field PIV technique.

Thermal dissatisfaction and draft effect were analyzed based on traditional pointwise measuring probes and the standards ISO 7730 and ASHRAE 55. It is revealed that thermal discomfort and draft sensation were substantially reduced using inserted lobes, without significant changes of pressure drop and sound pressure levels.

\section{KEYWORDS}

Multi-cone diffuser, inserted lobes, radial jet, PPD index, DR index 


\section{INTRODUCTION}

In mixing ventilation the airflow pattern in the occupied zone is highly dependent on the geometry of the diffuser. A high induction performance of the generated jet allows its optimal mix with indoor air, such that occupants would be more satisfied in terms of thermal comfort and air quality. In summer conditions, air jet is introduced with a lower temperature than the ambience. Buoyancy force accelerate the flow, and the resulting cold air dumping in the occupied zone may generate a localized draft discomfort for occupants in slightly cool to neutral thermal states. To minimize this problem, the air is generally supplied such that the Coanda effect may occur to attach the jet to the ceiling or a wall [1, 2]. Most radial diffusers are based on that principle. Their internal geometry can be arranged to produce either a vertical pattern (in heating mode) or a horizontal pattern (in cooling mode) so that the jet attaches to the ceiling and spreads radially along its surface. As a consequence, the entrainment is reduced compared to the corresponding free jet, since it takes place on only one side of the jet, increasing axial jet velocity by a factor of $\sqrt{2}[1,3]$. Among radial diffusers available on the market, the multi-cone diffuser [4-9] is widely used in commercial and office spaces. Another popular ceiling-mount diffuser is the vortex diffuser [4-7, 10], that produces a jet with a high degree of spin about the axis parallel to its main direction.

Koestel [3] was probably the first to conduct an analysis of jet patterns from different radial diffusers. The author pointed the importance of the deflection angle $(\Phi)$ between the horizontal and the jet outlet. About ten years after, Becher [11] has provided interesting information and practical recommendations on different types of air diffusers, among them the multi-cone diffuser. The decrease in the velocity of the air jet in the "third zone" [1] is modeled in isothermal conditions by the following equation:

$$
U_{\max } / U_{0}=C \sqrt{R_{0} \cdot S \cdot \cos (\Phi) /\left(X\left(X-R_{0}\right)\right)}
$$

Where $C$ is the velocity decay coefficient, $U_{\max }$, the jet centerline velocity at a distance $X$ from the diffuser, and $U_{0}$, the initial outlet velocity. This equation takes into account the deflection angle $\Phi$, the thickness of the slot $S$ (or $S_{\text {outer }}$ the normal distance between the two outer cones), and the size of the diffuser (horizontal distance $R_{0}$ between the neck centerline of the diffuser and its outer trailing edge). When the radial diffuser is mounted flush with the ceiling, the supplied air tends to form a jet downwards with $\Phi>30^{\circ}$. With $\Phi<30^{\circ}$, the jet attaches to the ceiling and flows radially along its surface.

Much later, Chuah et al. [4] conducted an experimental study under isothermal conditions to analyze jets issued from three flush-mounted ceiling diffusers: a vortex diffuser was 
compared to two multi-cone diffusers, one composed of three cones having, from the inner to the outer cone, $\Phi=56,45$ and $53^{\circ}$, and the other was composed of six cones having a constant angle $\Phi=45^{\circ}$. In contrast to the vortex diffuser, the multi-cone diffusers do not include a control disk of air diffusion pattern. The authors have shown that the three-cone diffuser produces a vertical jet, whereas the six-cone diffuser produces a horizontal ceilingattached jet. The authors were interested in induction performance of jets. They have based their analysis on the decrease in jet velocity in the third zone, given by the law:

$$
U_{\max } / U_{n}=K_{v}\left(\sqrt{A_{n}} / X\right)
$$

In this equation, which is widely used in the literature $[1,4,5,12], K_{v}$ is the velocity decay coefficient and is directly related to the entrainment level of the jet. The reference values considered are diffuser neck velocity $\left(U_{n}\right)$ and diffuser neck-area $\left(A_{n}\right)$, and $U_{\max }$ is the air speed captured by omnidirectional hot-wire anemometer. In their analysis, the authors have omitted the jet from the three-cone diffuser because it was not ceiling-attached jet. Larger $K_{v}$ values were obtained for the vortex diffuser and were attributed to the swirl effect. In the same decade, Shakerin and Miller [5] have conducted a similar study, by comparing in isothermal conditions three types of vortex diffusers to a three-cone diffuser with unknown deflection angles. The authors obtained slightly higher $K_{v}$ values for vortex diffusers and concluded that they generate a little more induction than the three-cone diffuser.

The obtained higher $K_{v}$ values for vortex diffusers in [4, 5] seem inconsistent with a widely accepted principle of $K_{v}$ decreasing with the increase of jet induction. In fact, there is a consensus in the literature $[1,3,6,10]$, that high entrainment implies a relatively small $K_{v}$ value. The inconsistency may be due to the inappropriate measurement technique used. Rather than the radial component $U_{\max }$, the air speed captured by omnidirectional anemometer is used to establish the jet velocity decay law.

$\mathrm{Hu}$ et al. [7] conducted an experimental investigation in cooling mode of flow pattern and thermal comfort generated by ceiling flush-mounted diffusers. A four-cone diffuser of $\boldsymbol{\Phi}=$ $48^{\circ}, 40^{\circ}, 30^{\circ}$ and $40^{\circ}$, respectively, is compared to a nozzle type diffuser and a vortex diffuser. The authors observed that most of the mixing of the air jet with ambient air took place in the vicinity of the diffuser outlet. As a consequence, both high mixing diffusers were able to provide a very good indoor thermal comfort environment compared with that provided by the multi-cone diffuser. It is to be noted that the multi-cone diffuser tested suffered from an early jet separation and a cold air dumping in the occupied zone. 
In the category of high induction diffusers, Nastase et al. [13] proposed an innovative concept, i.e. the lobed diffuser. In isothermal conditions the authors compared jet pattern and thermal comfort using wall-mounted grille with undulated fins to a reference grille with straight fins. The results demonstrate better induction and thermal comfort using lobed diffuser without additional pressure losses and noise. The authors attributed the greater induction of the lobed diffuser to their ability to produce large scale streamwise vortices at the inlet due to the imprint of the undulated trailing edge of the diffuser. Actually, the authors conducted academic fluid mechanics studies on elementary lobed jets for the characterization of the phenomena at the origin of the entrainment increase, in comparison to reference round jets [14-18].

Main diffuser design parameters are the room characteristic length $(L)$, the jet throw $\left(L_{T}\right)$, and the jet separation distance $\left(x_{S}\right)$ [10]. $L$ represents the horizontal length to which the jet needs to extend. $L_{T}$ is defined as the distance from the diffuser outlet to the point where the jet centerline velocity has decreased to a specified terminal velocity $V_{t}[2,6,10] . V_{t}=0.25 \mathrm{~m} / \mathrm{s}$ is usually selected, but other values have also been used [7, 8, 10, 19]. Considering $V_{t}=0.25$ $\mathrm{m} / \mathrm{s}$, the recommended throw ratio $L_{T} / L$ for circular ceiling diffusers in cooling mode is from 0.8 to 1.5 [10]. When the ceiling diffuser is properly designed, the jet has tendency to attach to the ceiling surface. However, it could detach from it if the downward buoyancy force is larger than the force exerted by the ambient pressure acting upward [10, 20]. When such separation occurs, the separation distance $\left(x_{S}\right)$ is defined as the distance from the diffuser outlet to the point where the jet separates from the ceiling [10, 21]. The above parameters influence the airflow pattern and the resulting indoor thermal conditions may affect the thermal comfort in the occupied zone.

In the literature, there is a real lack of coupling information between the airflow pattern generated by a radial jet and associated thermal comfort in non-isothermal conditions. There is also a lack of strategies for enhancing the jet induction in mixing ventilation, especially in the cooling season when draft risk could be important. This paper presents an experimental investigation of air distribution patterns and thermal comfort level generated inside a climate chamber by a radial jet issued from a conventional ceiling flush-mounted multi-cone diffuser. The climate chamber is used to simulate summer conditions under steady state thermal conditions. The impact on jet behavior and the resulting thermal comfort due to inserted lobes [22] as induction promoters into the multi-cone diffuser are evaluated. 


\section{EXPERIMENTAL APPARATUS}

\subsection{Test chamber and air handling circuit}

The experiments were carried out in a cubic laboratory chamber of an edge length of 3470 mm, coupled to an air diffusion circuit including an air handling unit (Fig. 1, a). The six inside faces are thermally controlled, using a hydraulic circuit composed of capillary tubes inserted in the walls and connected to a heat pump. To simulate a standard volume of residential or office building, a dropped ceiling was installed at height $2500 \mathrm{~mm}$. The mean ambient temperature $\left(T_{a}\right)$ at the extraction (see Fig. 1, a) is controlled with the hydraulic circuit. The jet flow is generated by the air handling unit equipped with a fan, a heater and a chiller, followed by a plenum box. The diffuser is installed at the extremity of the plenum box. The initial flow rate $\left(Q_{0}\right)$ and the air temperature $\left(T_{0}\right)$ are controlled with sensors placed between the handling unit and the plenum box, and again measured at the jet exit using a balometer from ACIN (Flowfinder mk2). The accuracy in flow rate measurement is $\pm 3 \%$ of the reading. Exit velocity profile from a diffuser depends greatly on the velocity distribution in the upstream neck. As the present study aims to quantify the effect of inserted lobes into a commercial diffuser, on the jet dynamics and the resulting thermal comfort, the neck velocity profile should be uniform, with low turbulence intensity. Hence, after the air handling unit, the air passes through a plenum box including a divergent, a short duct equipped with a perforated plate normal to the duct, a convergent, and again a short duct equipped with a honeycomb (Fig. 1, a and b). The round jet generated by the plenum box without the diffuser has been characterized in isothermal conditions. Near the exit, at $Z / D=0.25$ (Fig. 1, b), the mean radial velocity profiles (Fig. 1, c) measured on two normal axes are similar and almost flat in the region of the jet core. The turbulent intensity profile (Fig. 1, d) is flat at about $2 \%$ in the central region. Thus, it is shown herein that the flow through the neck is uniform, axisymmetric a priori, and very weakly turbulent.

\section{INSERT FIGURE 1 HERE}

A simplified seated manikin (Fig. 2) of 8 heated parts [23] and a total power of 81W, was used to simulate an occupant located at the center of the room (Fig. 4). The manikin was 
designed to have a mean skin temperature of $32{ }^{\circ} \mathrm{C}$ in a room with ambient temperature at $26^{\circ} \mathrm{C}$ and with still air.

\section{INSERT FIGURE 2 HERE}

\subsection{Diffusers}

A three-cone circular ceiling-mounted diffuser (Fig. 3, a-c) of constant angle $\Phi=33^{\circ}$, having adjustable cones, was chosen and will be designated by "conventional diffuser” (CD). The two inner cones can be adjusted manually (Fig. 3, c) to switch from vertical jet behavior to radial jet behavior. The present study is conducted in cooling mode, hence, the diffuser is set as shown in Fig. 3, c (right) to generate a radial jet. When equipped with inserted lobes as shown in Fig. 3, d-e, the diffuser is designated by "lobed diffuser" (LD). Compared to the "built-in lobed diffuser" of Nastase et al. [13], the proposed concept of inserted lobes [22] allows an easier integration of jet passive control into commercial diffusers without changes in their manufacturing process. The jet passive control is obtained by vortex promoters introduced into the conventional diffuser without intrinsic changes of its geometry.

\section{INSERT FIGURE 3 HERE}

The geometric parameters of the inserted lobes are given in Fig. 3, f-g. They were made of resin material and were built by a 3D printer. They have a thickness of $1 \mathrm{~mm}$ (Fig. 3, f), and have an undulated trailing edge length of $1164 \mathrm{~mm}$ and $2436 \mathrm{~mm}$, for the inner and the outer insert, respectively, leading to an obstruction ratio of about $10 \%$ of the diffuser effective area

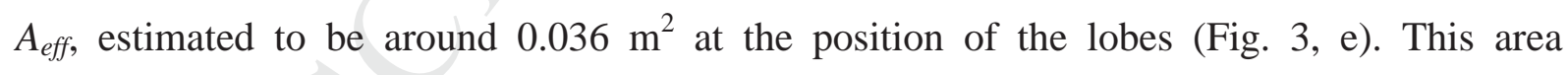
corresponds to the sum of areas of inner and outer slots at the location of inserted lobes, of thickness $S$ ( $S_{\text {inner }} \sim 31 \mathrm{~mm}$; $S_{\text {outer }} \sim 38 \mathrm{~mm}$ ) and perimeter $p t$, which is equal to inserted lobe perimeter (see Fig. 3, d). It is to be noted that the $A_{\text {eff }}$ of the multi-cone diffuser considered (Fig. 3, a and b) is not provided by the manufacturer, and could vary with the setting of the cones (Fig. 3, c) and the point where one define the diffuser outlet. This fact explains in the present study the choice of the neck-area $A_{n}\left(A_{n}=\pi D^{2} / 4\right.$, where $D=160 \mathrm{~mm}$ is the diffuser neck-diameter), as a reference parameter, like this has been done in [4]. The corresponding neck velocity $W_{n}=Q_{0} / A_{n}$, will be used as the reference velocity. 


\subsection{PIV measurements}

According to the review of Cao et al. [24], nowadays with current advanced laser and camera technologies, Particle Image Velocimetry (PIV) becomes an ideal measurement tool to investigate the airflow pattern generated in indoor spaces.

In this study, two PIV measurement campaigns were conducted using the same 2D2C Dantec Dynamics PIV system of $2 \mathrm{~Hz}$ acquisition rate. It is composed of a Dantec HiSense 11M CCD camera with a sensor of $4000 \times 2672$ pixels, and a dual-cavity $200 \mathrm{~mJ}$ laser. The airflow is seeded with olive oil droplets generated by a laskin nozzle. The CCD camera is equipped with a Nikkor $50 \mathrm{~mm}$ lens for large scale PIV measurements (LS-PIV) and with a Sigma $105 \mathrm{~mm}$ macro lens for small scale PIV measurements (SS-PIV). The LS-PIV aims towards a global investigation of the flow in the half middle cross section $(1900 \times 2100 \mathrm{~mm})$ of the room (green color in Fig. 4, a), resulting from the jet and its interaction with the heated manikin. A total of 8 PIV regions of $1000 \times 670 \mathrm{~mm}$ in size each were assembled to construct the whole field. The SS-PIV aims towards a local investigation of the jet. In particular, the maximum velocity of the boundary-layer near the ceiling which was not captured in LS-PIV, is obtained with SSPIV. A total of 4 PIV regions of $374 \times 139 \mathrm{~mm}$ each (red color in Fig. 4, a) were assembled to construct the ceiling jet field of $1274 \times 139 \mathrm{~mm}$ in size. For each PIV region, 600 and 1000 pairs of images for LS-PIV and SS-PIV, respectively, were acquired and processed through an adaptive multi-grid correlation algorithm handling the sub-pixel window displacement. The final size of the interrogation window was $64 \times 64$ pixels and $32 \times 32$ pixels, respectively, with an overlap of $50 \%$. The resulting vector spacing are $8.4 \times 8.4 \mathrm{~mm}$ and $1.5 \times 1.5 \mathrm{~mm}$ for global field and jet field, respectively.

\section{INSERT FIGURE 4 HERE}

The accuracy of PIV measurements could be influenced by many parameters. Most of the error sources have been discussed by Raffel et al. [25]. It consists mainly in systematic errors due among them to, correlation method, background noise, seeding density and size of particles. According to Sandberg [26], in ventilated rooms the velocity is low and the relative systematic errors for a 2D-PIV measurement could be estimated to be around 1 to $2 \%$. The contribution of insufficient particle size to this error (i.e. peak locking effect) could be higher for LS-PIV measurements using oil droplets. This effect has been systematically checked in 
the present study by computing displacement histograms. No peak locking effect was detected due to high accuracy sub-pixels interpolation algorithm.

\subsection{Thermal comfort evaluation}

To evaluate the thermal discomfort, standards ISO 7730 [27] and ASHRAE 55 [28] have adopted the PMV-PPD model (Predicted Mean Vote-Predicted Percentage of Dissatisfied) and the DR index (Draft Rate), proposed by Fanger [29, 30]. The PMV index was originally developed for human subjects in homogenous thermal environmental conditions and the corresponding PPD is the percentage of people dissatisfied by the thermal environment. According to Fanger [29] and Awbi [31], for a uniform thermal environment, a single value of PMV-PPD indices is sufficient to express the thermal discomfort in the occupied zone. For non-uniform thermal environment, the authors proposed that measurements should be carried out at various points in the occupied zone and these are then used to calculate the distribution of PMV-PPD indices throughout the zone [31]. Although there is a dispute regarding the use of PMV-PPD model in non-uniform environmental conditions, this model was considered in such conditions in several studies, among them those of Wan and Chao [32] and Arghand et al. [33]. It is used by the authors in both global and local evaluation of thermal comfort generated by ventilation systems.

The DR index is a local discomfort model which characterizes draft effect on the neck of an occupant and may be extended to other body regions [31]. Nastase et al. [13], Chow and Wong [34], and Tomasi et al. [35] have used this index to study experimentally the thermal comfort generated by mixing ventilation diffusers.

In this study, thermal comfort was investigated using the method proposed by Fanger [29] for non-uniform environments. Environmental parameters such as, air temperature and air speed were measured using thermocouples (type $\mathrm{K}$, accuracy of $\pm 0.3{ }^{\circ} \mathrm{C}$ ) and hot-sphere anemometers (TSI 8475, accuracy of $\pm 3 \%$ of the reading). The occupied zone was meshed with 16 vertical canes (Fig. 4, b), each including 4 sensors located at $0.1 \mathrm{~m}, 0.6 \mathrm{~m}, 1.2 \mathrm{~m}$ and $1.8 \mathrm{~m}$ relatively to the floor. These positions correspond to the levels of ankles, waist, head of a seated occupant and head of a standing occupant [28, 29], respectively. Wall-surface temperatures $\left(T_{\mathrm{p}}\right)$ measurements were also performed. Each wall including the floor and the ceiling is divided into 4 subzones of equal dimensions, each one equipped with a thermocouple located in its center. Each wall-surface has almost uniform temperature with a maximum difference of $1.3{ }^{\circ} \mathrm{C}$ between the 4 corresponding subzones. For the entire test 
chamber, the maximum difference is $2.2^{\circ} \mathrm{C}$. For mean radiant temperature $\left(\overline{T_{r}}\right)$ calculation, the method described in [29] and ISO 7726 [36] based on wall-surface temperatures of the 6 faces and a seated manikin with unknown azimuthal angle is applied.

For each experiment, the duration to achieve steady-state conditions is about 6 hours. After this period, environmental parameters measurements were carried out for a minimum duration of 8 hours with a sampling time of 1 minute. Personal parameters such as metabolic rate, mechanical work and clothing insulation, were fixed at 1.2 met, $0 \mathrm{~W} / \mathrm{m}^{2}$ and 0.7 clo respectively, to evaluate the thermal comfort of a seated occupant, wearing a working clothing ensemble and having a sedentary activity. Calculations of thermal comfort indices were performed with a home-made software and the obtained PMV-PPD values were verified using the ASHRAE 55 Comfort tool [28].

\subsection{Static pressure and sound pressure level measurements}

The total pressure loss was measured for the two diffusers according to EN 12238 standard [37] using a KIMO MP110 micro-manometer. One pressure tap is installed at $1.5 \mathrm{D}$ upstream the diffuser and the second in the occupied zone outside the jet region.

Sound pressure levels were recorded with and without the diffusers using a handheld type 2250 Sound Level Meter of Class 1 Precision (tolerance of $\pm 0.7 \mathrm{~dB}$ ) from Bruel \& Kjaer. According to ISO 10052 [38], a diffuser is considered as a technical equipment, and $L_{\text {eq }}$ (dBA) must be measured. $L_{\text {eq }}$ is the time average equivalent sound pressure level with Aweighting. The averaging time is $6 \mathrm{~s}$ as specified in [39] and measurements were performed as recommended in [38] at two locations P1 and P2 (see Fig.1, a). These positions are corresponding respectively to the nearest position to the diffuser located in the occupied zone of the room, and the limit of the occupied zone in the case where people are seated.

\subsection{Tested configurations}

The multi-cone diffuser considered (Fig.3, a-c) is recommended for flow rates ranging from 200 to $400 \mathrm{~m}^{3} / \mathrm{h}$. The air handling unit equipped with its plenum box (Fig. $1 \mathrm{a}-\mathrm{b}$ ) and the multi-cone diffuser, provides flow-rates in the range of 15 to $325 \mathrm{~m}^{3} / \mathrm{h}$. Hence, in this study the tested volumetric flow-rate were set to $Q_{0}=200 \mathrm{~m}^{3} / \mathrm{h}$ and $Q_{0}=300 \mathrm{~m}^{3} / \mathrm{h}$. The operating conditions are summarized in Table 1 and Table 2. Thermal comfort measurements were carried out for these two volumetric flow rates, considering a cold jet (CJ) with a difference between ambient air temperature $\left(T_{a}\right)$ and supply air temperature $\left(T_{0}\right)$ of $\Delta T=10^{\circ} \mathrm{C}$. Thermal 
comfort evaluation was performed in the cases referenced as CD_CJ_HM and LD_CJ_HM (Table 1), with respectively CD and LD (Fig. 3) in the presence of the heated manikin (HM). In this study, the operative temperature $\left(T_{o p}\right)$ was estimated as the mean value of the mean air temperature $\left(\bar{T}_{\text {air }}\right)$ and the mean radiant temperature $\left(\bar{T}_{r}\right)$, under the specified condition of mean air speed ( $\bar{V}_{\text {air }}<0.2 \mathrm{~m} / \mathrm{s}$, see ISO 7726 standard [36]).

LS-PIV and SS-PIV measurements were conducted for CD_CJ_HM and LD_CJ_HM, when the supply flow rate is $Q_{0}=200 \mathrm{~m}^{3} / \mathrm{h}$ (Table 2).

To study the influence of the heated manikin on one hand, and of jet buoyancy force on the other hand, on the flow pattern in the room, two supplementary cases were considered, i.e., CD_CJ, which refers to the cold jet, and CD_IJ, which refers to the isothermal jet issuing from the CD without manikin (Table 2).

The SS-PIV campaign was conducted to capture the jet features. For this campaign, in addition to the foregoing cases, the experiments were conducted for two additional cases using LD, referenced LD_CJ and LD_IJ, for cooling and isothermal conditions respectively (Table 2). The flow control parameters used in this study are the jet Reynolds number (Re) and the jet Archimedes number (Ar). They are defined below and reported in Table 1 and Table 2.

$$
\begin{aligned}
& \mathrm{Re}=W_{n} \sqrt{A_{n}} / \nu \\
& A r=g \sqrt{A_{n}} \Delta T / T_{a} W_{n}^{2}
\end{aligned}
$$

Where $v$ is the cinematic viscosity of the air and $\mathrm{g}$ is the gravitational acceleration.

The cooling load $Q_{t}$ (Eq. 5) is defined as the thermal energy that must be removed from the space in order to maintain the desired comfort conditions. This quantity is reported in Table 1 and Table 2 for the different cases considered.

$$
Q_{t}=\rho C_{p} Q_{b} \Delta T / S_{f}
$$

Where $\rho$ is the air density, $C_{p}$ the air specific heat and $S_{f}$ the floor surface.

INSERT TABLE 1 HERE

INSERT TABLE 2 HERE

\section{RESULTS AND DISCUSSION}




\subsection{Global and local analysis of air flow pattern}

Velocity distributions from LS-PIV in the streamwise median plane of the room, are given in Fig. 5, a1, b1, c and d, for CD_CJ_HM, LD_CJ_HM, CD_CJ and CD_IJ respectively.

\section{INSERT FIGURE 5 HERE}

Considering the reference cases CD_CJ_HM and LD_CJ_HM, similarities and differences appear in the global airflow patterns. The air distribution is a result of several coupled effects: (i) jet confinement and its interaction with the walls, (ii) negatively buoyancy effect which leads to jet acceleration towards the occupied zone and (iii) interaction of the jet with the manikin, which is both a solid obstacle and a source of a thermal plume.

The recirculation zone appearing outside the jet is due to parallel opposite flows, which are the downward jet and the upward flow above the manikin. This ascending flow may be the result of cumulative effects: the thermal plume and the diffuser suction, heightened by the manikin as a solid obstacle which opposes to the jet development in the CD case. The velocity in the jet from LD decreases faster, so that the jet interacts weakly with the manikin. Consequently, the recirculation behavior is different in the LD case (with one recirculation zone) than in the CD case (with a double recirculation zone).

The near ceiling region airflow patterns from SS-PIV are provided in Fig. 5, a2 and b2 for CD_CJ_HM and LD_CJ_HM cases. As visible in these figures, the jet issuing from each diffuser attaches to the ceiling due to the Coanda effect.

When approaching the ceiling-wall corner, the jet separates from the ceiling due to the damping effect exerted by the opposing wall and spreads downwards along the wall after impingement on it. The cold jet (Fig. 5, a1, b1 and c) separates from the wall at $x_{S}=\left(L+Z_{S}\right)$, where $L=1735 \mathrm{~mm}$ is the room characteristic length. For either CD or LD, $x_{S} / L$ is greater than unity (Table 3) because the ceiling radial jet turns downward at the ceiling/wall corner. The recommendation $X_{S} / L>1$ given in [10] to prevent that cold air draft enters the occupied zone is met. $X_{S} / L$ for CD_CJ_HM, LD_CJ_HM, and CD_CJ are 1.75, 1.46 and 1.60, respectively. The reduction of about $17 \%$ in $X_{S}$ value in the LD_CJ_HM relatively to the CD_CJ_HM, is due to high induction generated by inserted lobes as revealed by the jet fields 
(Fig. 5, a2 and b2). The 9\% difference between $x_{S}$ values of CD_CJ_HM and CD_CJ is due to the heated manikin. The presence of the heated manikin results in two opposite effects on the separation distance: (i) the thermal plume from the manikin would tend to heat faster the jet flow, which would generate its early separation from the wall, and (ii) the confinement of the jet generated by the manikin as a solid obstacle, has the effect of redressing the jet streamlines and flatten the jet against the wall, leading to an increase of the separation distance. The second effect seems predominant relatively to the first one.

Jet separation from the wall occurs because the jet velocity becomes low and the temperature difference between the jet and the local ambient air becomes positive. In the isothermal case CD_IJ (Fig. 5, d), the vertical jet do not separate from the wall, confirming the previous explanation.

The throw $L_{T}$ of the jet is also reported in Table 3 using different values of terminal velocity, i.e., $0.1,0.25$ and $0.37 \mathrm{~m} / \mathrm{s}$. $V_{t}=0.37 \mathrm{~m} / \mathrm{s}$ is chosen for comparison to the manufacturer value [19] obtained using CD in isothermal conditions for $Q_{0}=200 \mathrm{~m}^{3} / \mathrm{h}$. The obtained value of $L_{T} / L$, ranging from 0.86 to 0.88 , is almost constant. The gap to the manufacturer's value

(0.81) is of 6 to $9 \%$, which is low. For $L D$, the obtained value $L_{T} / L=0.60$ is lower due to the higher entrainment of the corresponding jet.

Considering $V_{t}=0.25 \mathrm{~m} / \mathrm{s}$, the recommended throw ratios $L_{T} / L$ for cooling applications in the case of circular ceiling diffusers, is from 0.8 to 1.5 [10]. All the obtained values for cooling cases (Table 3 ) fall in the recommended range. The isothermal case CD_IJ with $L_{T} / L=1.58$ exceeds by $5 \%$ the recommended upper limit. The effect of buoyancy force and heated manikin are rather low when compared to the effect of the inserted lobes. Anyway, the obtained value $L_{T} / L=0.80$ for LD_CJ_HM remains in the recommended range.

The gap in $L_{T} / L$ values between CD_CJ_HM and LD_CJ_HM is not constant since it rises to $46 \%$ when $V_{t}=0.25 \mathrm{~m} / \mathrm{s}$ and falls to $21 \%$ for $V_{t}=0.10 \mathrm{~m} / \mathrm{s}$. This observation suggests that the effect of high induction of the lobed jet on the decrease of its axial velocity, tends to be weaker far away from the jet inlet, relatively to its counterpart non lobed jet. This effect was already noted in both academic work [15] and HVAC related work [13] of lobed jets.

\section{INSERT TABLE 3 HERE}

The flow above the manikin (Fig. 6 a and b) was extracted from Fig. 5 a and b. The corresponding velocity profiles at two stations are compared (Fig. 6, c and d) with those 
obtained at the same stations by Borges et al. [40], above a humanlike seated thermal manikin placed in still air and almost isothermal conditions $\left(T_{o p}=19{ }^{\circ} \mathrm{C}\right)$. In [40], air speed measurements were performed using omnidirectional probes whereas in the present study, the third component not captured by LS-PIV is neglected. In the LD_CJ_HM, the velocity profile is slightly narrower than in CD_CJ_HM, which could be explained by the higher air sucking generated by the lobes and by lower velocities in the occupied zone. The obtained velocity profiles are clearly influenced by the surrounding flow from the jet since they are wider to those of [40] obtained in surrounding still air. The gap in the position of maximum velocity between the simplified and the humanlike manikins could be explained by their respective geometry [41]. As for the gap on velocity magnitude relative to Borges et al. [40] data, which is about $20 \%$ in the worst case, it is difficult to conclude on the actual reason. Apart from the effect of the geometry of the manikin, in the present study the third component not measured is neglected, while the authors directly measure the air speed. Also, the manikin of the author is thermally controlled and placed in an environment with $T_{\text {op }}=19{ }^{\circ} \mathrm{C}$, while ours has a constant power per zone in an environment with different value of $T_{\text {op }}$ (see Table 1 ). Anyway, as reported in the literature [42, 43], a simplified manikin is sufficient when the interest deals with the global flow pattern in the occupied zone.

\section{INSERT FIGURE 6 HERE}

Fig. 7, a-d gives the radial velocity $(U)$ and the vertical velocity $(W)$ profiles extracted from SS-PIV velocity fields (Fig. 5, a2, b2). The two shear layers corresponding to the two blowing slots are deflected in CD towards the ceiling and merge rapidly. No trace of the double-shear layer is visible on the jet radial velocity profiles (Fig. 7, a). In the LD, the lobes render persistent the double shear-layer (Fig. 6, b2), as confirmed by the corresponding radial velocity profiles (Fig. 7, b). Both jets become ceiling-attached between $X / \sqrt{A_{n}}=1.3$ and 1.6 (Fig. 7, a, b). However, as revealed by $W$-profiles at $X / \sqrt{A_{n}}=1.3$ (Fig. 7, c, d), the depression is much lower in the jet from LD than that in the jet from CD because of larger air induction. Beginning with $X / \sqrt{A_{n}}=4.2$ (Fig. 7, e, f), the radial velocity profiles are self-similar in both jets (Fig. 7, g, h), and are in a good agreement with similarity laws of two-dimensional walljets given in [12] and [44]. 
The jet half velocity width $Z_{0.5}$ (Fig. 8, a) is also in good agreement with the literature law of a two-dimensional wall-jet [12] when considering the region $X / \sqrt{A_{n}} \geq 3.5$ in each jet. The obtained coefficient $(0.066)$ is close to the value $(0.068)$ given in [12]. The jet width $Z_{0.1}$ defined as the distance $Z$ from the ceiling where the radial velocity is 0.1 from its maximum value $U_{\max }$ is also linear (Fig. 8, b). In the jet near field ( $X / \sqrt{A_{n}}<3.5$ ), the larger expansion of the jet from LD compared to the one from CD (Fig. 8, a, b) is due to higher entrainment of ambient air and faster diffusion of jet momentum.

Maximum jet velocity $\left(U_{\max }\right)$ evolution is displayed in two different representations (Fig. 8, c, d) in the region $1<X / \sqrt{A_{n}}<16$. The first one (Fig. 8, c) refers to Eq. 1 proposed by Becher [11]. Good agreement is observed for $X / \sqrt{A_{n}}>4$ with the recommended law for CD. As expected, $U_{\max }$ decreases faster in the jet from LD. One do not observe significant effect of inlet temperature $T_{0}$.

The second representation (Fig. 8, d) refers to Eq. 6 suitable for isothermal or non-isothermal horizontal jets $[1,4,5,12]$, where $\rho_{0}$ is air density at the jet exit and $\rho_{a}$, density of the ambient air. The data of $[4,5]$ are included in Fig. 8, d. The velocity in the jet from the vortex diffuser should decrease faster than that in the jet from multi-cone diffuser. The authors in [4, 5] used the air speed to establish the jet velocity decay law, rather than the maximum radial velocity component $U_{\max }$. As the jet from the vortex diffuser is highly rotational, the use of the air speed undoubtedly explains the above inconsistency. In the case of multi-cone diffusers, vertical and transverse velocity components at the position of $U_{\max }$ are null. Hence, the consistency between our CD's data with those of $[4,5]$ is not surprising.

$\frac{U_{\max }}{W_{n}}=K_{v}\left(\frac{\sqrt{A_{0}}}{X+X_{0}}\right)\left(\frac{\rho_{0}}{\rho_{a}}\right)^{0.5}$

Neglecting the virtual origin by putting $X_{0}=0$ in Eq. $6, \mathrm{~K}_{\mathrm{v}}$ values of the present study are summarized in Table 4. The heated manikin and/or buoyancy force generate on the $\mathrm{K}_{\mathrm{v}}$-value a low deviation, less or equal to $4 \%$ relatively to the isothermal case. In contrast, the mean deviation in terms of $\mathrm{K}_{\mathrm{v}}$-value of $\mathrm{LD}$ cases compared to CD cases is about $25 \%$. This difference is due to the higher ambient air entrainment in the lobed jet. 


\section{INSERT TABLE 4 HERE}

\subsection{Thermal comfort analysis}

The DR index includes the turbulence intensity which is not accessible using a hot-sphere anemometer. The standard ISO 7730 [27] recommends in this case a value of $40 \%$. A maximum difference of $0.1 \%$ in DR distributions is recorded, using respectively this recommended value and turbulence intensity (ranging from 9 to $12 \%$ ) measured by PIV in the occupied zone. Hence, the value of $40 \%$ has been adopted in all cases.

Fig. 9 shows the statistic distribution of the 64 nodes in terms of thermal comfort levels (A, B, C) provided in ISO 7730 standard [27]. These levels fix minimum values of PPD and DR indices (Table 5).

\section{INSERT FIGURE 9 HERE}

\section{INSERT TABLE 5 HERE}

Based on the distribution of these levels, the higher thermal comfort is achieved using the LD. For $Q_{s}=200 \mathrm{~m}^{3} / \mathrm{h}$, all points have reached the higher level $\mathrm{A}$ in terms of PPD and DR indices using LD. For $Q_{s}=300 \mathrm{~m}^{3} / \mathrm{h}$, the indices show a marked degradation of the thermal comfort. However, the LD performance relative to CD is more visible in this case. In terms of DR index, for LD 87\% of data falls into the category A, compared to 53\% for CD. The PPD index is also improved using inserted lobes, with $6 \%$ of data falling outside the range A-C in LD compared to $31 \%$ in CD. Furthermore, $85 \%$ of data falls into the range A-B for LD compared to $41 \%$ in $\mathrm{CD}$.

In Fig. 10 are displayed the distributions of PPD and DR indices in the occupied zone. The distributions are organized in 4 vertical planes, each one corresponding to an interpolation of 16 measurement points. Low comfort level regions are located near the corners of the room. From Fig. 11, a-d, which is providing the corresponding distributions of velocity magnitude 
and air temperature, it seems that the increase of velocity near the corners is responsible for the degradation of the comfort in these regions.

\section{INSERT FIGURE 10 HERE}

The distance between the diffuser centerline and the end wall (Fig. 11, e) has the minimum value along the normal direction $\left(L_{n}=L=1.74 \mathrm{~m}\right)$ and the maximum value along the diagonal direction $\left(L_{d}=2.44 \mathrm{~m}\right)$. The radial jet, after its traveling along the ceiling, impinges on the vertical walls (Fig. 11, e), with an inclination angle $0 \leq \theta \leq 45^{\circ}$, relatively to the axis normal to the wall. It is well known that an impinging jet on an inclined wall (relatively to the jet direction), generates on the wall two asymmetric radial wall flows: the downhill flow and the uphill flow, the former being more intense and larger than the latter [45]. The confluence of downhill flows towards the corners causes recirculation bubbles being at the origin of high local velocities (Fig. 11, a, b) and high PPD and DR indices (Fig. 10) in the occupied zone. This is less critical when the diffuser is with (LD), than without inserted lobes (CD).

\section{INSERT FIGURE 11 HERE}

\subsection{Acoustic and pressure loss data analysis}

Fig. 12, a shows total pressure losses for CD and LD, measured following EN 12238 standard [37], as a function of the inlet volumetric flow rate $Q_{0}$. The two diffusers display similar values of the pressure losses, with differences less than the accuracy of the pressure sensor $( \pm 2 \mathrm{~Pa})$.

Fig. 12, b and c presents at positions P1 and P2 (Fig. 1, a), global sound pressure levels as a function of the initial volumetric flow rate $Q_{0}$. These global sound pressure levels reflect in the same time the possible noise generation of the diffusers and of the air handling unit and air distribution ducts. The air diffusion system is equipped with a plenum box (Fig. 1, b), on which the diffuser is mounted. Three cases were considered: in the first one the measurements were performed without any diffuser mounted on the plenum, and the other two cases correspond to the operation of the system with CD and LD. In the proximity of the diffuser (position P2) it could be observed from Fig. 12, c that for the lowest initial volumetric flow rates $\left(150 \mathrm{~m}^{3} / \mathrm{h}\right.$ and $\left.175 \mathrm{~m}^{3} / \mathrm{h}\right)$, the global sound pressure level displays higher levels in the case without diffuser. This means that for small values of volumetric flow rates, both diffusers 
are responsible for a part of the attenuation of the noise generated by the air handling unit and ducts. For these data points, the contribution of the diffusers themselves on the global noise generation can not be extracted directly.

For larger values of initial flow rate $\left(Q_{0} \geq 200 \mathrm{~m}^{3} / \mathrm{h}\right)$, the sound pressure levels of the diffusers themselves were extracted (Fig. 12, d). As recommended by ISO 10052 standard [38], mean values of pressure levels were calculated as a representative values of global noise generated in the occupied zone (Fig. 12, e). The mean value is an average weighted by the coefficients 2/3 and 1/3 of sound pressure levels in P1 and P2, respectively.

For $Q_{0}=200 \mathrm{~m}^{3} / \mathrm{h}$ one could notice a slight difference in the mean value of $L_{e q}$ with an advantage for LD. For $Q_{0}>200 \mathrm{~m}^{3} / \mathrm{h}$, the supplementary noise generated by the presence of inserted lobes is in average of $2 \mathrm{~dB}(\mathrm{~A})$.

The sound pressure levels in the occupied zone (Fig. 12, b and c) with each diffuser are under $45 \mathrm{~dB}(\mathrm{~A})$ which is acceptable for office spaces, according to national legislations and building codes in most of European countries [46].

\section{INSERT FIGURE 12 HERE}

\section{CONCLUSION}

Jet characteristics, indoor airflow pattern, and thermal comfort were analyzed in a full-scale room model in mixing ventilation and cooling mode. An occupant has been simulated using a seated simplified shape heated manikin positioned at the center of the room.

The ventilating jet is generated from a multi-cone diffuser (CD), flush-mounted in the center of the ceiling and operating in radial jet generation. The effect of inserted lobes introduced into the diffuser being LD has been evaluated.

The depression generated by the Coanda effect is much stronger in the jet from CD than that in the jet from LD. The depression is lower in LD because of larger air induction and faster diffusion of jet momentum at the vicinity of the diffuser outlet. The higher entrainment of ambient air produces a larger jet expansion and a faster decrease of jet velocity. Without inserted lobes, the decrease of jet velocity from the multi-cone diffuser is consistent with available data and laws of the literature.

In the far field, both jets from CD and LD, display a two dimensional wall-jet behavior in accordance with the corresponding laws of literature. 
Thermal comfort analysis based on both ASHRAE 55 and ISO 7730 standards, reveals significant improvement in the LD case compared to the CD case. This is achieved without significant increase in pressure drop and noise. In the two cases, low comfort level zones were located near the corners of the room, due to high velocity magnitude in these regions. The confluence of downhill flows towards the corners in the wake of radial jet impingement on the walls, causes recirculation bubbles being the origin of high local velocities and high local discomfort. This is less critical when the diffuser is with lobed inserts than without.

\section{ACKNOWLEDGEMENT}

This work was supported by the grants of the French National Research Agency (ANR) as part of the FLUBAT- ANR-12-VBDU-0010 project.

\section{REFERENCES}

[1] ASHRAE, Space air diffusion, in ASHRAE Handbook: Fundamentals, ASHRAE, Editor. 2009.

[2] Cao, G., Awbi, H., Yao, R., Fan, Y., Sirén, K., Kosonen, R., and Zhang, J., A review of the performance of different ventilation and airflow distribution systems in buildings. Building and Environment, 2014. 73: p. 171-186.

[3] Koestel, A., Jet velocities from radial flow outlets, in Semi-annual Meeting of ASHACE, ASHRAE, Editor. 1957: Murray Bay Canada.

[4] Chuah, Y.K., Hu, S.C., and Barber, J.M., Airflow Characteristics of Circular Ceiling Diffusers. International Journal on Architectural Science, 2000. 1: p. 59-67.

[5] Shakerin, S. and Miller, P.L. Experimental study of Vortex Diffusers. in 1996 Annual Conference. 1996. San Antonio, TX.

[6] Aziz, M.A., Gad, I.A.M., Mohammed, E.S.F.A., and Mohammed, R.H., Experimental and Numerical Study of A/C Outletsand Its Impact on Room Airflow Characteristics. World Academy of Science, Engineering and Technology, 2012. 6: p. 387-396.

[7] Hu, S.C., Barber, J.M., and Chiang, H., Full-scale Thermal Performance Tests of Alternative Diffusers when operating with Cold Air, in Ashrae Transactions. 1999.

[8] Shioya, M., Sagara, N., Miura, K., Tanabe, S., Tsubota, Y., and Yanagihara, R., Thermal comfort aspects of cold air distribution system, in Ashrae Transactions. 1995.

[9] Miller, P.L. and Nevins, R.G., Room air distribution performance of ventilating ceilings and cone-type circular ceiling diffusers, in Ashrae Transactions. 1970.

[10] Kirkpatrick, A.T. and Elleson, J.S., Selection of cold air diffusers, A. Transaction, Editor. 1998.

[11] Becher, P., Air distribution in ventilated rooms, in Heating and air-technical Conference. 1966, JIHVE: Budapest. p. 219-227.

[12] Rajaratnam, N., Turbulent jets. 1976, Amsterdam, Netherlands: Elsevier Scientific Publishing Company.

[13] Nastase, I., Meslem, A., Vlad, I., and Colda, I., Lobed grilles for high mixing ventilation - An experimental analysis in a full scale model room. Building and Environment, 2011. 46(3): p. 547-555. 
[14] Nastase, I., Meslem, A., and Gervais, P., Primary and secondary vortical structures contribution in the entrainement of low Reynolds number jet flows. Experiments in Fluids, 2008. 44(6): p. 1027-1033.

[15] Nastase, I. and Meslem, A., Vortex dynamics and mass entrainment in turbulent lobed jets with and without lobe deflection angles. Experiments in Fluids, 2010. 48(4): p. 693-714.

[16] El Hassan, M. and Meslem, A., Time-resolved stereoscopic particle image velocimetry investigation of the entrainment in the near field of circular and daisy-shaped orifice jets. Physics of Fluids, 2010. 22(3): p. 035107.

[17] El Hassan, M., Meslem, A., and Abed-Meraim, K., Experimental investigation of the flow in the near-field of a cross-shaped orifice jet. Physics of Fluids, 2011. 23(4): p. 045101.

[18] Meslem, A., El-Hassan, M., and Nastase, I., Analysis of jet entrainment mechanism in the transitional regime by time-resolved PIV. Journal of Visualization, 2011. 14(1): p. 41-52.

[19] ALDES. Documentation technique diffuseur série AF/AT 842. Available from: http://pro.aldes.fr/html/grilles-et-diffusion-d-air-diffuseurs-plafonniers-serie-af-842af-842-thermo-aluminium.htm\#description.

[20] Hassani, V., Malmstrom, T., and Kirkpatrick, A.T., Indoor thermal environment of cold air distribution systems, A. Transaction, Editor. 1993.

[21] Adre, N. and Albright L, D., Criterion for establishing similar two-dimensional wall jet airflow patterns in confined spaces., A. Transactions, Editor. 1994.

[22] Vialle, P.J., Leroy, E., and Meslem, A., Diffusion device with lobe inserts and fan coil comprising such a device, in European Patent Office. EP2933575. October 2015.

[23] Dogeanu, A., Iatan, A., Bode, F., Croitoru, C., and Nastase, I., Conception of a simplified seated thermal manikin for CFD validation purposes. Romanian Journal of Civil Engineering, 2014. 5.

[24] Cao, X., Liu, J., Jiang, N., and Chen, Q., Particle image velocimetry measurement of indoor airflow field: A review of the technologies and applications. Energy and Buildings, 2014. 69(0): p. 367-380.

[25] Raffel, M., Willert, C.E., Wereley, S.T., and Kompenhans, J., Particle Image Velocimetry A Practical Guide. Second Edition ed. 2007.

[26] Sandberg, M., Whole-Field Measuring Methods in Ventilated Rooms. HVAC\&R Research, 2007. 13(6): p. 951-970.

[27] ISO, Ergonomics of the thermal environment -- Analytical determination and interpretation of thermal comfort using calculation of the PMV and PPD indices and local thermal comfort criteria, in ISO 7730. 2005.

[28] ANSI/ASHRAE, Thermal environmental conditions for human occupancy, in Standard 55-2013. 2013.

[29] Fanger, P.O., Thermal Comfort, Analysis and Applications in Environmental Engineering. 1970: Mc Graw Hill Book Company.

[30] Fanger, P.O., Melikov, A.K., Hanzawa, H., and Ring, J., Air turbulence and sensation of draught. Energy and Buildings, 1988. 12(1): p. 21-39.

[31] Awbi, H.B., Ventilation of Buildings. Second edition ed. 2003, London, U.K.: SPON. 522.

[32] Wan, M.P. and Chao, C.Y., Experimental Study of Thermal Comfort in an Office Environment with an Underfloor Ventilation System. Indoor and Built Environment, 2002. 11(5): p. 250-265.

[33] Arghand, T., Karimipanah, T., Awbi, H.B., Cehlin, M., Larsson, U., and Linden, E., An experimental investigation of the flow and comfort parameters for under-floor, 
confluent jets and mixing ventilation systems in an open-plan office. Building and Environment, 2015. 92: p. 48-60.

[34] Chow, W.K. and Wong, L.T., Experimental studies on air diffusion of a linear diffuser and associated thermal comfort indices in an air-conditioned space. Building and Environment, 1994. 29(4): p. 523-530.

[35] Tomasi, R., Krajčík, M., Simone, A., and Olesen, B.W., Experimental evaluation of air distribution in mechanically ventilated residential rooms: Thermal comfort and ventilation effectiveness. Energy and Buildings, 2013. 60: p. 28-37.

[36] ISO, Ergonomics of the thermal environment -- Instruments for measuring physical quantities, in ISO 7726. 1998.

[37] AFNOR, Ventilation des bâtiments - Bouches d'air - Essais aérodynamiques et caractérisation pour applications en diffusion à mélange, in NF EN 12238. 2001.

[38] ISO, Acoustique -- Mesurages in situ de l'isolement aux bruits aériens et de la transmission des bruits de choc ainsi que du bruit des équipements -- Méthode de contrôle, in ISO 10052. 2004.

[39] Ministère de l'Écologie du Développement durable et de l'Énergie Ministère du Logement et de l’Égalité des territoires, Guide de Mesures Acoustiques. 2014.

[40] Borges, C.M., Gaspar, A.R., and Quintela, D.A. Analysis of the interaction of thermal plumes within office environment using a thermal manikin. in 7th International Thermal Manikin and Modelling Meeting. 2008. University of Coimbra.

[41] Zukowska-Tejsen, D., Melikov, A., and Popiolek, Z., Impact of geometry of a sedentary occupant simulator on the generated thermal plume. H V A C \& $\mathrm{R}$ Research, 2012. 18(4): p. 795-811.

[42] Topp, C., Hesselholt, P., Trier, M.R., and Nielsen, P.V. Influence of geometry of thermal manikins on room airflow. in Healthy Buildings 2003 - Proceedings 7th International Conference. 2003. National University of Singapore.

[43] Zukowska, T.D., Melikov, A.K., and Popiolek, Z. Thermal plume above a simulated sitting person with different complexity of body geometry. in Roomvent 2007: Proceedings of the 10th International Conference on Air Distribution in Rooms. 2007. Helsinki, Finland.

[44] Verhoff, A., The two-dimensional turbulent wall jet with and without an external stream, P. University, Editor. 1963.

[45] Vipat, O., Feng, S.S., Kim, T., Pradeep, A.M., and Lu, T.J., Asymmetric entrainment effect on the local surface temperature of a flat plate heated by an obliquely impinging two-dimensional jet. International Journal of Heat and Mass Transfer, 2009. 52(2122): p. 5250-5257.

[46] Brelih, N., Thermal and acoustic comfort requirements in European standards and national regulations. REHVA Journal, 2013. 


\section{ACCEPTED MANUSCRIPT}

Table 1: Experimental conditions for thermal comfort evaluation

\begin{tabular}{|c|c|c|c|c|c|c|c|c|c|c|}
\hline \multirow[b]{2}{*}{ Cases } & \multicolumn{8}{|c|}{ Experimental conditions ${ }^{(*)}$} & \multirow[b]{2}{*}{$\mathrm{Ar}$} & \multirow[b]{2}{*}{$\operatorname{Re}$} \\
\hline & $\begin{array}{c}Q_{0} \\
{\left[\mathrm{~m}^{3} / \mathrm{h}\right]}\end{array}$ & $\begin{array}{c}T_{0} \\
{\left[{ }^{\circ} \mathrm{C}\right]}\end{array}$ & $\begin{array}{c}T_{a} \\
{\left[{ }^{\circ} \mathrm{C}\right]}\end{array}$ & $\begin{array}{c}\overline{T_{r}} \\
{\left[{ }^{\circ} \mathrm{C}\right]}\end{array}$ & $\begin{array}{c}T_{o p} \\
{\left[{ }^{\circ} \mathrm{C}\right]}\end{array}$ & $\begin{array}{l}\overline{T_{p}} \\
{\left[{ }^{\circ} \mathrm{C}\right]}\end{array}$ & $\begin{array}{l}R H \\
{[\%]}\end{array}$ & $\begin{array}{c}\mathrm{Q}_{\mathrm{t}} \\
{\left[\mathrm{W} / \mathrm{m}^{2}\right]}\end{array}$ & & \\
\hline \multirow{4}{*}{ CD_CJ_HM } & 200 & 13.4 & 23.4 & 24.3 & 23.8 & 24.7 & 42 & \multirow{2}{*}{55.8} & \multirow{2}{*}{0.0054} & \multirow{2}{*}{25000} \\
\hline & \pm 6 & \pm 0.2 & \pm 0.5 & \pm 0.8 & \pm 0.6 & \pm 0.8 & \pm 2 & & & \\
\hline & 300 & 13.4 & 22.3 & 23.2 & 22.4 & 22.7 & 48 & \multirow{2}{*}{74.5} & \multirow{2}{*}{0.0046} & \multirow{2}{*}{37600} \\
\hline & \pm 8 & \pm 0.2 & \pm 0.4 & \pm 0.7 & \pm 0.4 & \pm 0.7 & \pm 2 & & & \\
\hline \multirow{4}{*}{ LD_CJ_HM } & 200 & 13.4 & 23.8 & 25.6 & 25.1 & 26.0 & 42 & \multirow{2}{*}{58.0} & \multirow{2}{*}{0.0055} & \multirow{2}{*}{25000} \\
\hline & \pm 6 & \pm 0.2 & \pm 0.4 & \pm 0.7 & \pm 0.5 & \pm 0.7 & \pm 2 & & & \\
\hline & 300 & 13.4 & 22.3 & 23.2 & 22.3 & 22.8 & 48 & \multirow{2}{*}{74.5} & \multirow{2}{*}{0.0047} & \multirow{2}{*}{37600} \\
\hline & \pm 8 & \pm 0.2 & \pm 0.4 & \pm 0.8 & \pm 0.5 & \pm 0.8 & \pm 2 & & & \\
\hline
\end{tabular}

$\left(^{*}\right)$ Data are given as the mean value \pm standard deviation 


\section{ACCEPTED MANUSCRIPT}

Table 2: Experimental conditions for PIV measurements

\begin{tabular}{|c|c|c|c|c|c|c|c|c|}
\hline \multirow[b]{2}{*}{ Cases } & \multicolumn{2}{|c|}{ PIV Campaign } & \multicolumn{4}{|c|}{ Experimental conditions ${ }^{(*)}$} & \multirow[b]{2}{*}{$\mathrm{Ar}$} & \multirow[b]{2}{*}{$\operatorname{Re}$} \\
\hline & LS-PIV & SS-PIV & $\begin{array}{c}Q_{0} \\
{\left[\mathrm{~m}^{3} / \mathrm{h}\right]}\end{array}$ & $\begin{array}{c}T_{0} \\
{\left[{ }^{\circ} \mathrm{C}\right]}\end{array}$ & $\begin{array}{c}T_{a} \\
{\left[{ }^{\circ} \mathrm{C}\right]}\end{array}$ & $\begin{array}{c}\mathrm{Q}_{\mathrm{t}} \\
{\left[\mathrm{W} / \mathrm{m}^{2}\right]}\end{array}$ & & \\
\hline CD_CJ_HM & $\checkmark$ & $\checkmark$ & $\begin{array}{c}200 \\
\pm 6\end{array}$ & $\begin{array}{l}13.4 \\
\pm 0.2\end{array}$ & $\begin{array}{l}23.4 \\
\pm 0.5\end{array}$ & 55.8 & 0.0054 & 25000 \\
\hline LD_CJ_HM & $\checkmark$ & $\checkmark$ & $\begin{array}{l}200 \\
\pm 6\end{array}$ & $\begin{array}{l}13.4 \\
\pm 0.2\end{array}$ & $\begin{array}{l}23.8 \\
\pm 0.4\end{array}$ & 58.0 & 0.0055 & 25000 \\
\hline CD_CJ & $\checkmark$ & $\checkmark$ & $\begin{array}{l}200 \\
\pm 6\end{array}$ & $\begin{array}{l}13.4 \\
\pm 0.2\end{array}$ & $\begin{array}{l}23.4 \\
\pm 0.5\end{array}$ & 55.8 & 0.0054 & 25000 \\
\hline CD_IJ & $\checkmark$ & $\checkmark$ & $\begin{array}{l}200 \\
\pm 6\end{array}$ & $\begin{array}{l}23.0 \\
\pm 0.2\end{array}$ & $\begin{array}{l}23.0 \\
\pm 0.5\end{array}$ & 0 & 0 & 25000 \\
\hline LD_CJ & $x$ & $\checkmark$ & $\begin{array}{l}200 \\
\pm 6\end{array}$ & $\begin{array}{l}13.4 \\
\pm 0.2\end{array}$ & $\begin{array}{l}23.8 \\
\pm 0.5\end{array}$ & 58.0 & 0.0055 & 25000 \\
\hline LD_IJ & $x$ & $\checkmark$ & $\begin{array}{c}200 \\
\pm 6\end{array}$ & $\begin{array}{l}23.0 \\
\pm 0.2\end{array}$ & $\begin{array}{l}23.0 \\
\pm 0.2\end{array}$ & 0 & 0 & 25000 \\
\hline
\end{tabular}

(*) Data are given as the mean value \pm standard deviation

CD: Conventional diffuser, LD: Conventional diffuser with inserted lobes, CJ_HM: Cold Jet with Hetad Manikin, IJ: Isothermal Jet 


\section{ACCEPTED MANUSCRIPT}

Table 3: Wall separation distance and jet throw

\begin{tabular}{ccccc}
\hline Cases & $x_{S} / L$ & $\begin{array}{c}L_{T} / L \\
(0.1 \mathrm{~m} / \mathrm{s})\end{array}$ & $\begin{array}{c}L_{T} / L \\
(0.25 \mathrm{~m} / \mathrm{s})\end{array}$ & $\begin{array}{c}L_{T} / L \\
(0.37 \mathrm{~m} / \mathrm{s})\end{array}$ \\
\hline CD_CJ_HM & $\mathbf{1 . 7 5}$ & $\mathbf{2 . 1 7}$ & $\mathbf{1 . 4 8}$ & $\mathbf{0 . 8 8}$ \\
LD_CJ_HM & $\mathbf{1 . 4 6}(\mathbf{1 7 \% )}$ & $\mathbf{1 . 7 2}(\mathbf{- 2 1} \mathbf{\% )}$ & $\mathbf{0 . 8 0} \mathbf{( - 4 6} \%)$ & $\mathbf{0 . 6 0} \mathbf{( - 3 2} \%)$ \\
CD_CJ & 1.60 & 1.93 & 1.50 & 0.87 \\
CD_IJ & - & 2.01 & 1.58 & 0.86 \\
CD Manufacturer & - & - & - & 0.81 \\
data & - & & & \\
\hline
\end{tabular}


Table 4: Velocity decay coefficient for the different cases in the range $3 \leq X / \sqrt{A_{n}} \leq 9$

\begin{tabular}{ccccc}
\hline Cases & $K_{v}$ & $\mathrm{R}^{2}$ & \multicolumn{2}{c}{ Deviation of $\mathrm{K}_{\mathrm{v}}(\%)$} \\
\hline CD_IJ & 1.62 & 0.986 & Ref. for CD cases & Ref. for LD cases \\
CD_CJ & 1.68 & 0.982 & 3.7 & - \\
CD_CJ_HM & 1.63 & 0.982 & 0.6 & - \\
\hline LD_IJ & 1.19 & 0.988 & Ref. for LD cases & 26.4 \\
LD_CJ & 1.24 & 0.993 & 4.2 & 23.5 \\
LD_CJ_HM & 1.20 & 0.997 & 0.8 & 25.9 \\
\hline
\end{tabular}




\section{ACCEPTED MANUSCRIPT}

Table 5: Statistic distribution of $P P D$ and $D R$ indices for the two diffusers

\begin{tabular}{cccccc}
\hline $\begin{array}{c}\text { Cat. ISO } \\
7730\end{array}$ & Conditions & $\begin{array}{c}\text { Conventional } \\
200 \mathrm{~m}^{3} / \mathrm{h}\end{array}$ & $\begin{array}{c}\text { Lobed } \\
200 \mathrm{~m}^{3} / \mathrm{h}\end{array}$ & $\begin{array}{c}\text { Conventional } \\
300 \mathrm{~m}^{3} / \mathrm{h}\end{array}$ & $\begin{array}{c}\text { Lobed } \\
300 \mathrm{~m}^{3} / \mathrm{h}\end{array}$ \\
\hline A & $D R<10 \%$ & 98 & 100 & 53 & 87 \\
B & $10<D R<20 \%$ & 2 & 0 & 47 & 13 \\
C & $20<D R<30 \%$ & 0 & 0 & 0 & 0 \\
\hline A & $P P D<6 \%$ & 81 & 100 & 0 & 0 \\
B & $6<P P D<10 \%$ & 19 & 0 & 31 & 78 \\
C & $10<P P D<15 \%$ & 0 & 0 & 31 & 8 \\
out & $P P D>15 \%$ & 0 & 0 & 38 & 4 \\
\hline
\end{tabular}


(a)

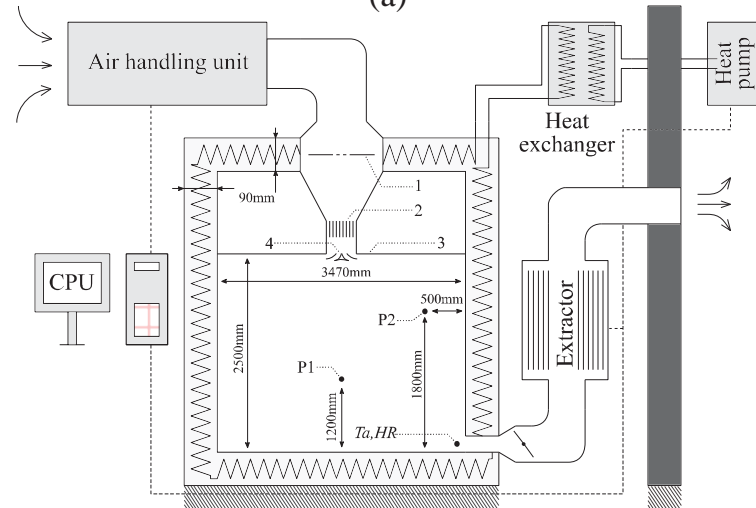

(c)

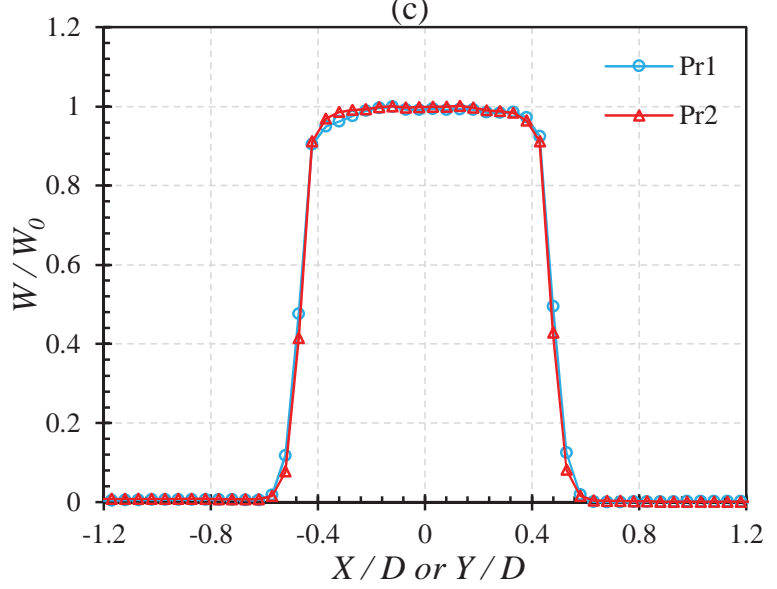

(b)

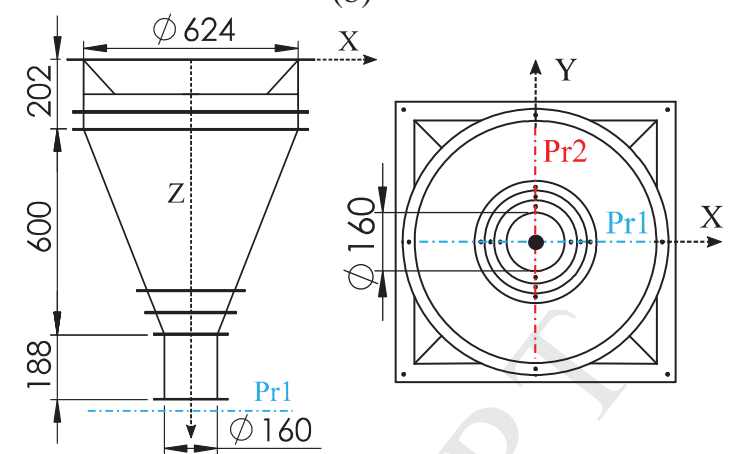

(d)

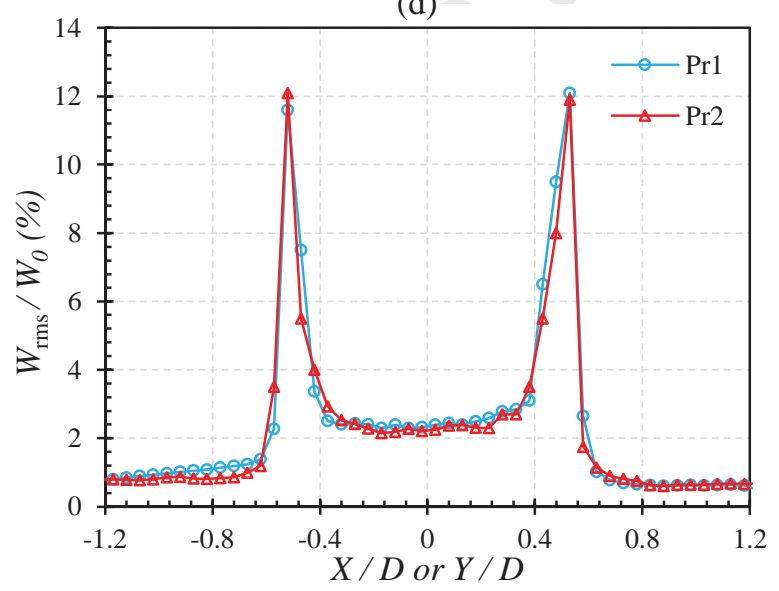

Fig. 1 (a) Sketch of the climate chamber - 1 perforated plate, 2 honeycomb, 3 diffuser, 4 dropped ceiling, P1 and P2 acoustic measurement points; (b) Details of the plenum box; (c) Mean velocity profile at the exit of the plenum box $(Z=0.25 D)$; (d) Turbulence intensity at the exit of the plenum box $(Z=0.25 D)$ with $D=160 \mathrm{~mm}$, the diameter of the neck of the plenum box and $W_{0}=2.13 \mathrm{~m} / \mathrm{s}$ the corresponding exit velocity 

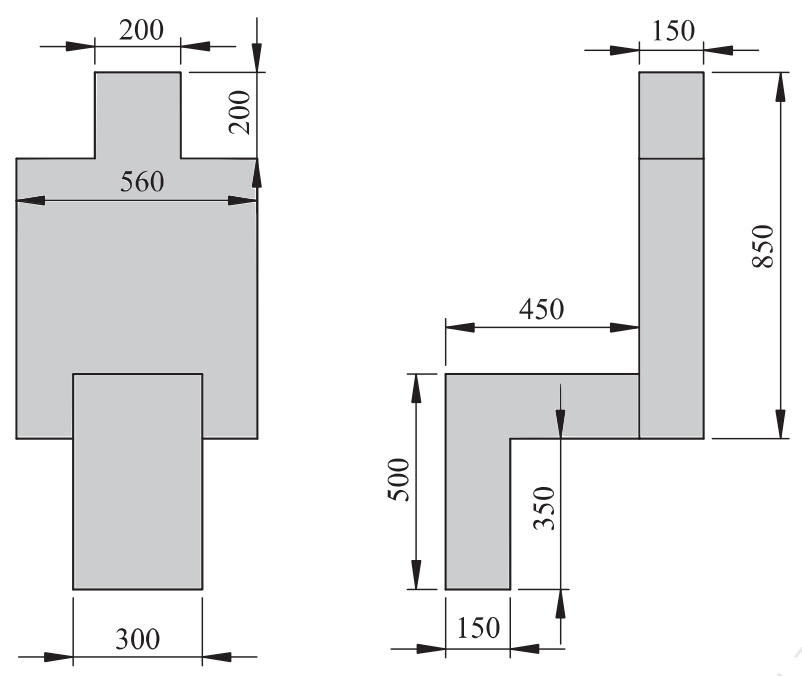

Fig. 2 Heated manikin - the dimensions are in $\mathrm{mm}$ 
(a)

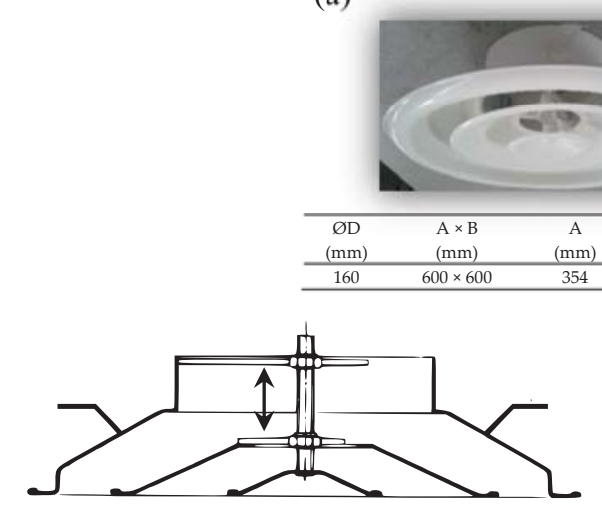

(d)

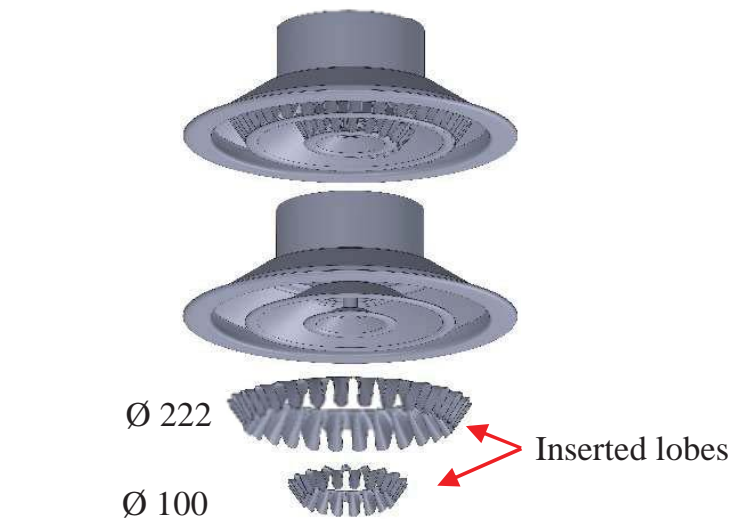

(f)

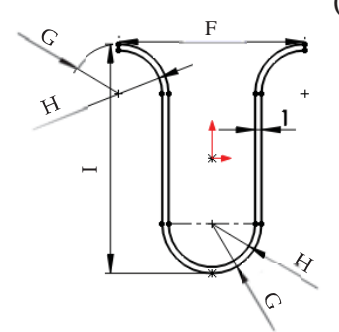

(b)

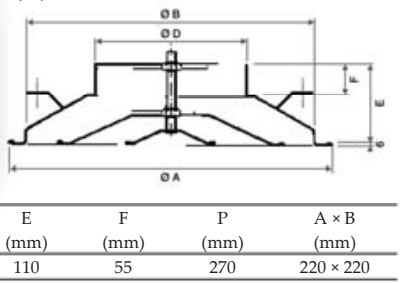

(c)

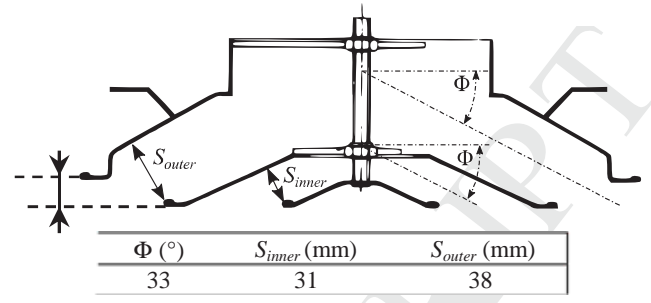

(e)

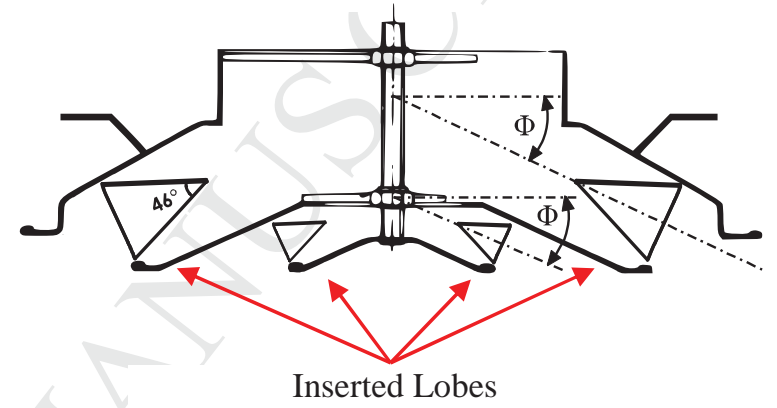

(g)

\begin{tabular}{cccccc}
\hline $\begin{array}{c}\text { Inserted } \\
\text { lobed }\end{array}$ & $\mathrm{F}$ & $\mathrm{G}$ & $\mathrm{H}$ & $\mathrm{I}$ & $\mathrm{J}$ \\
\hline$\varnothing 100$ & 22 & 6.0 & 5.0 & 27 & 24.01 \\
$\varnothing 222$ & 29.30 & 7.83 & 6.83 & 36.12 & 42.65 \\
\hline
\end{tabular}

Dimensions in $\mathrm{mm}$

Fig. 3 (a, b) Conventional diffuser (CD) and its dimensions; (c) Control-setting of jet behavior - double-headed arrow (left) indicates the movement up/down of the inner cones, the two arrows (right) indicate the position of inner cones for radial jet generation; (d, e) Mounting of the inserted lobes into the CD, (f, g) Geometry details of the inserted lobes 
(a)

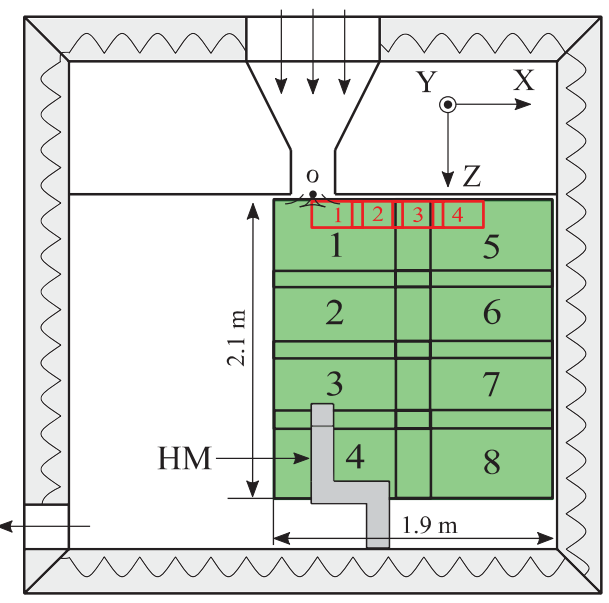

(b)

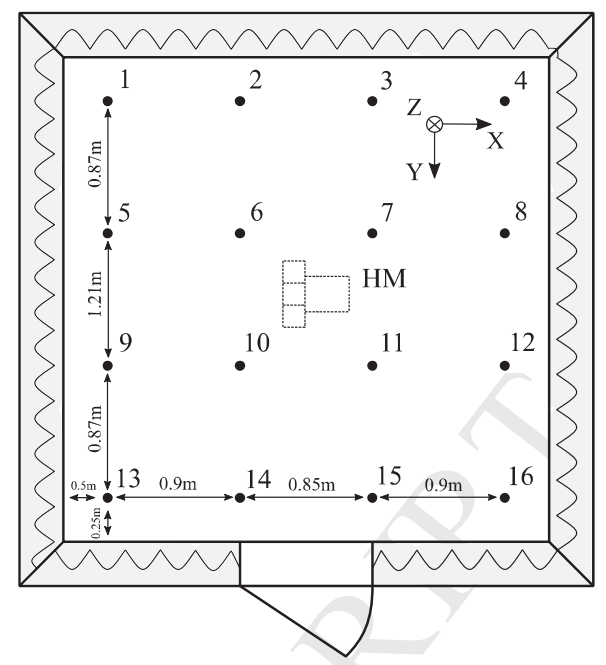

Fig. 4 (a) PIV windows in the vertical half middle cross section of the room (LS-PIV in green) and in the corresponding jet region (SS-PIV in red); (b) positions in the horizontal plane of the room of the heated manikin (HM), and of 16 verticals canes of hot-sphere anemometers and thermocouples 


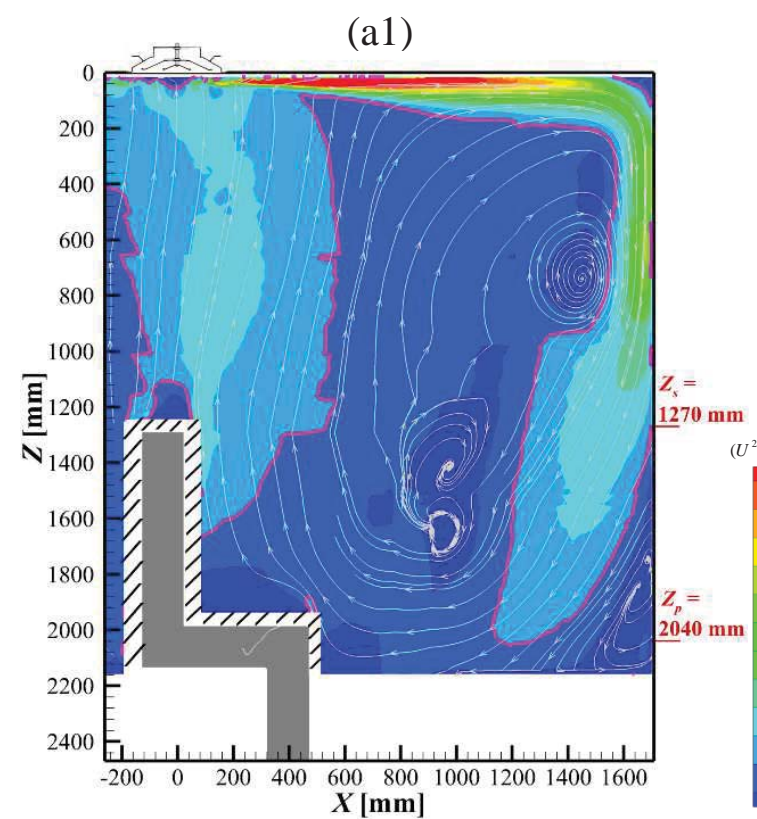

(a2)

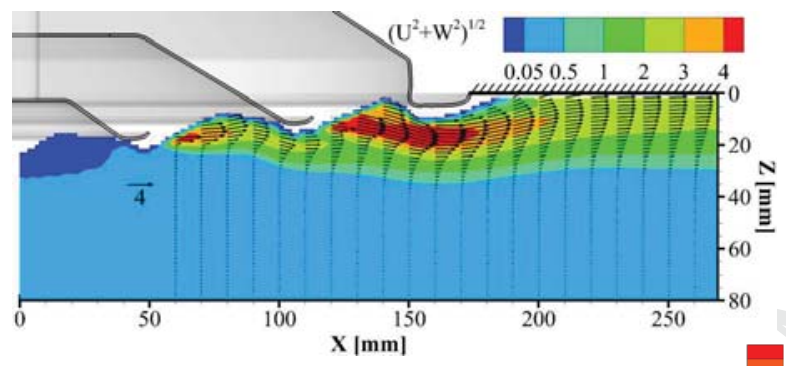

(c)

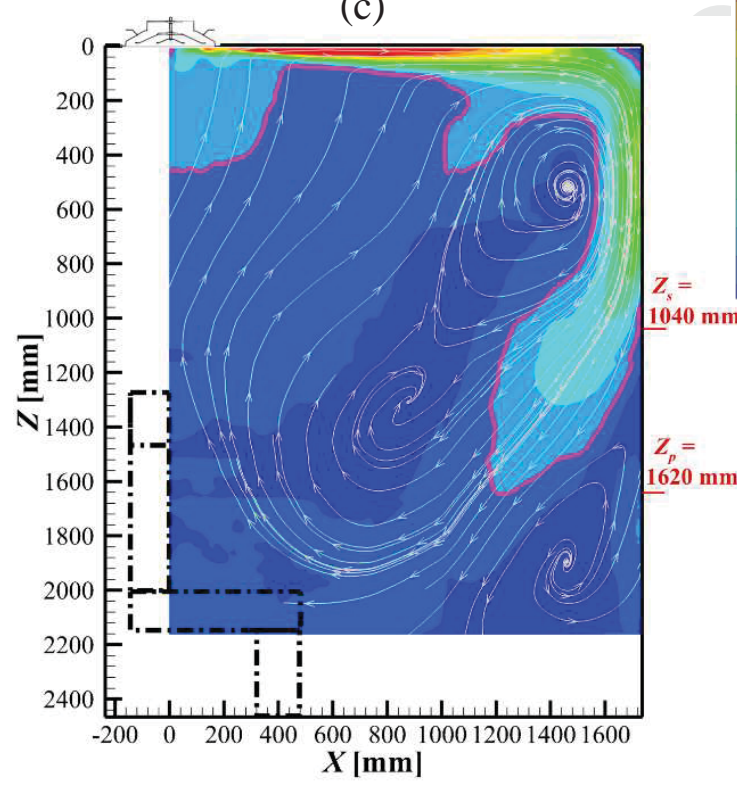

(b1)

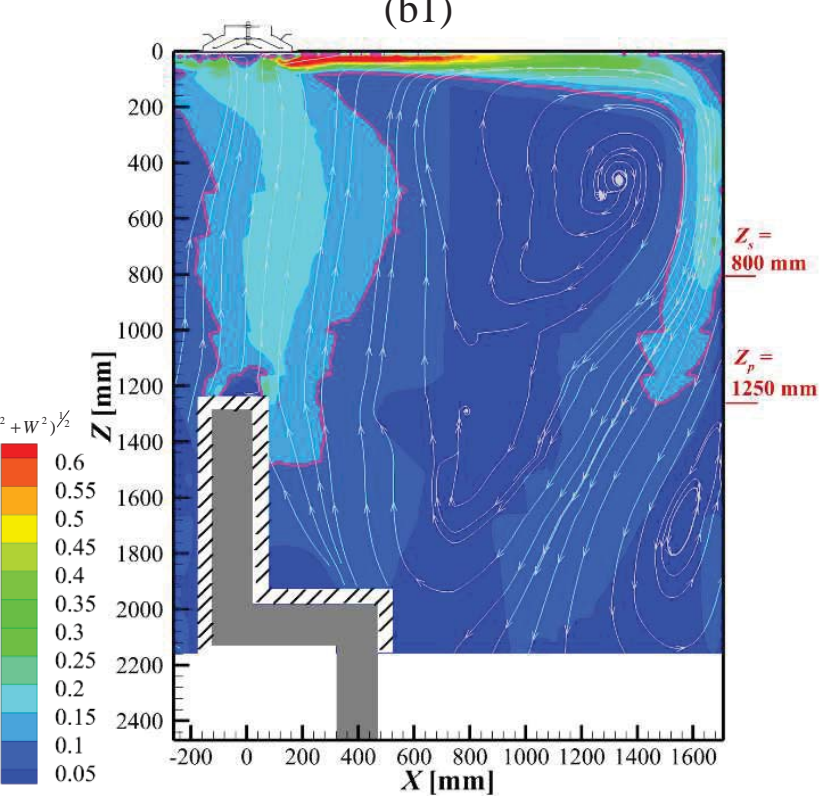

(b2)

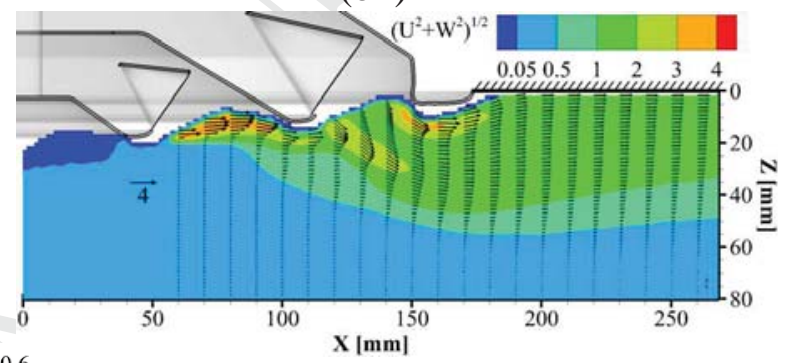

(d)

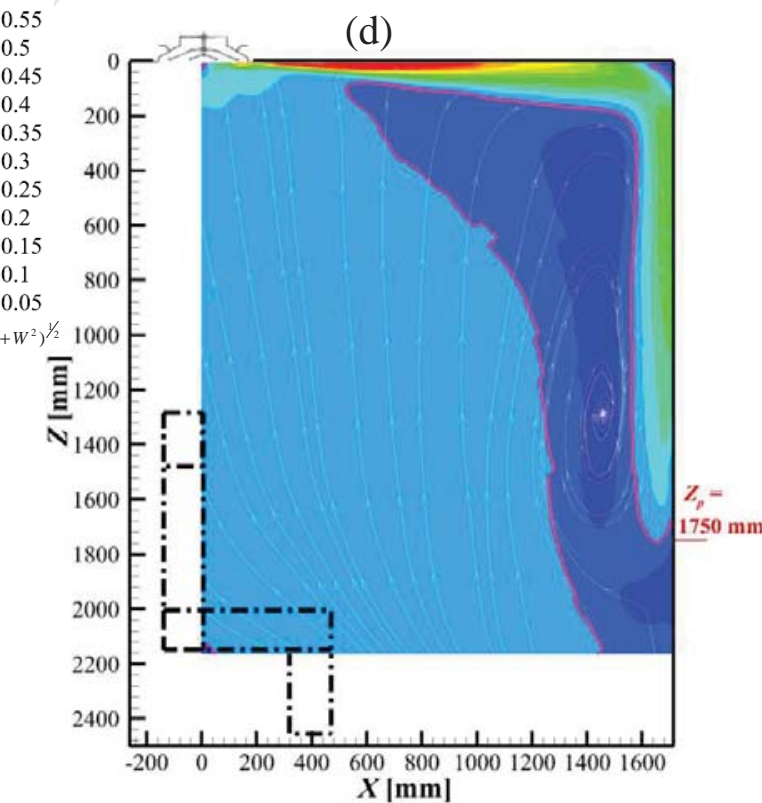

Fig. 5 Isocolors of velocity magnitude for $Q_{0}=200 \mathrm{~m}^{3} / \mathrm{h}$ in the half middle cross section of the room,: (a1) CD_CJ_HM, (b1) LD_CJ_HM, (c) CD_CJ, (d) CD_IJ - hatching zone around the manikin corresponds to the laser scattering, the magenta line represents $0.1 \mathrm{~m} / \mathrm{s}$ isocontour; (a2, b2) Isocolors of velocity magnitude and vector profiles in the ceiling jet region for cases 


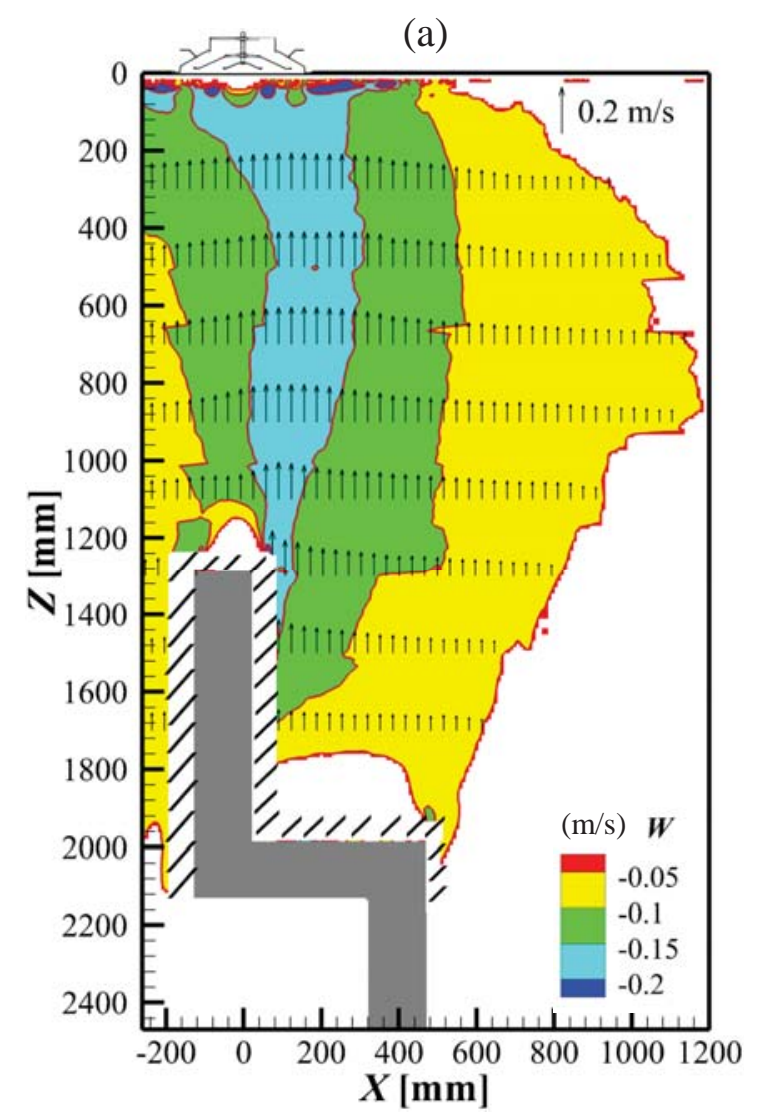

(c)

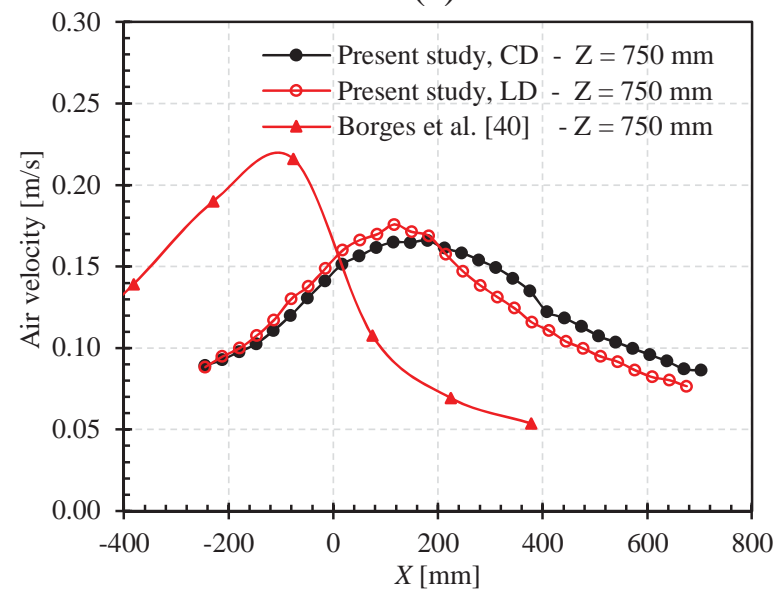

(b)

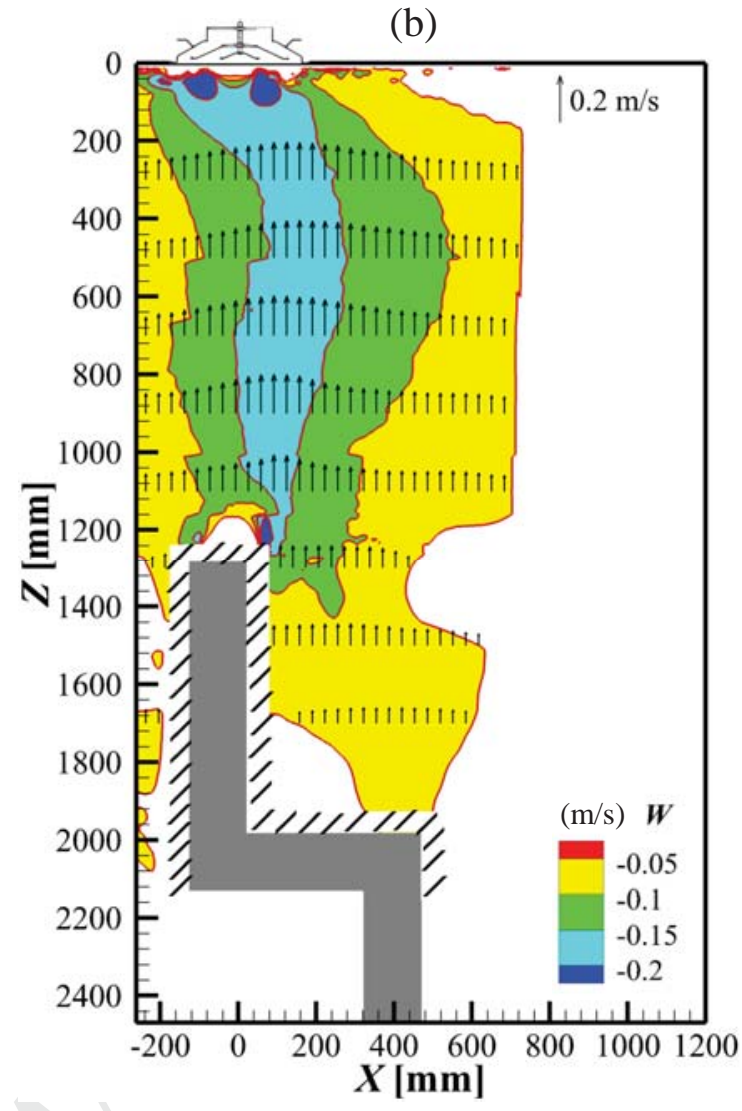

(d)

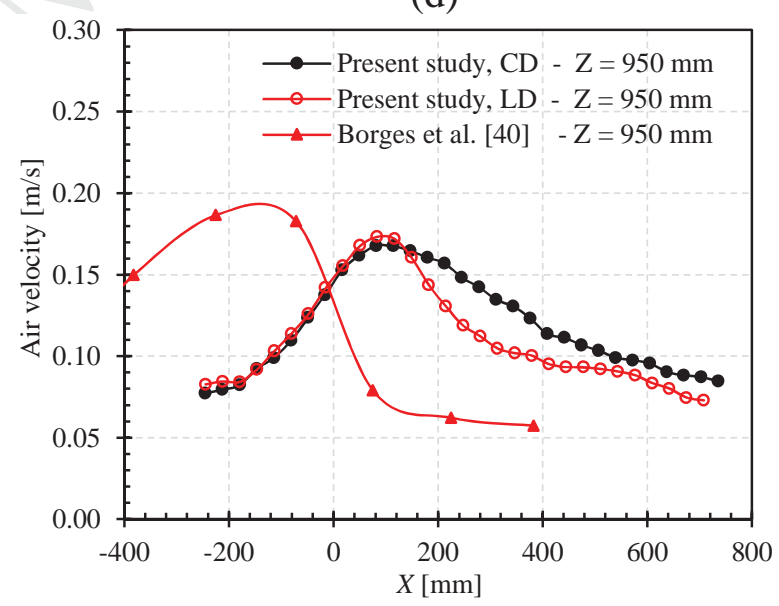

Fig. 6 (a, b) Axial velocity ( $W$ ) contours (color map) and profiles (vectors) around the heated manikin: (a) CD_CJ_HM, (b) LD_CJ_HM; (c, d) Air velocity profiles above the manikin Comparison with the literature [40] 
(a)

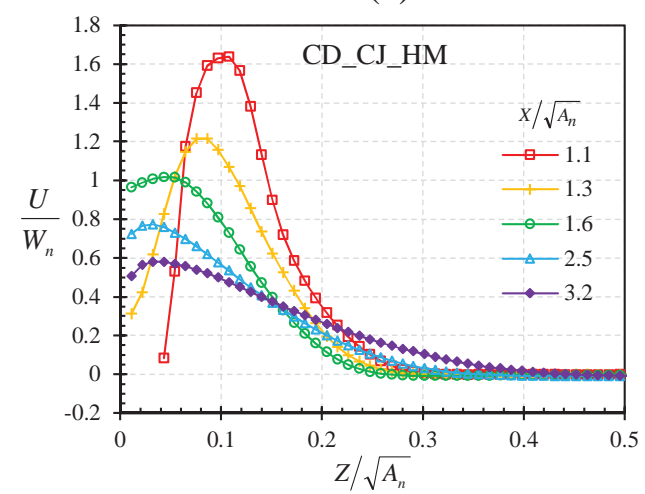

(c)

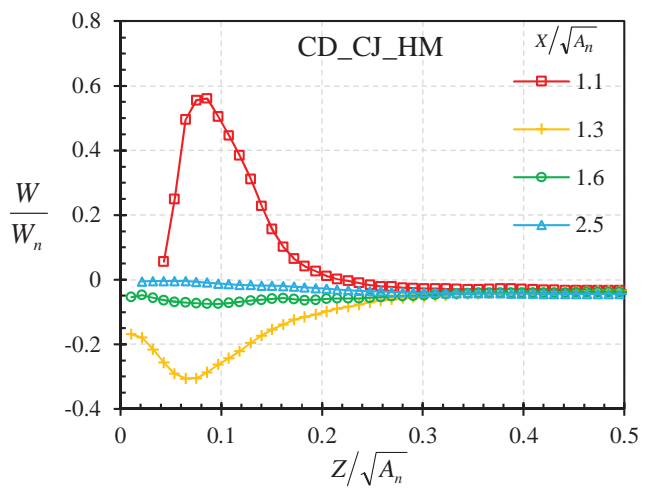

(e)

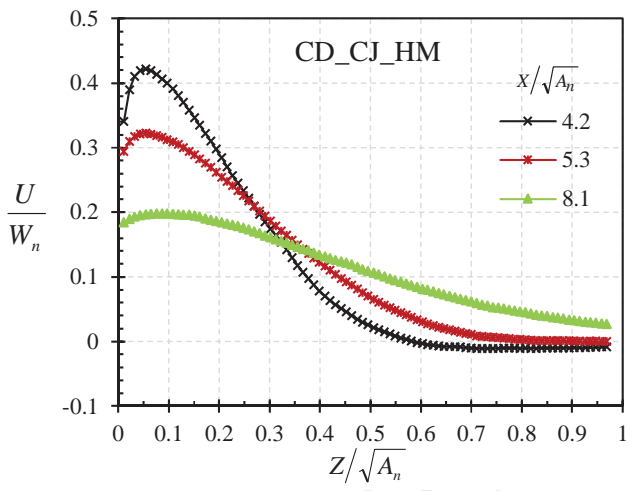

(g)

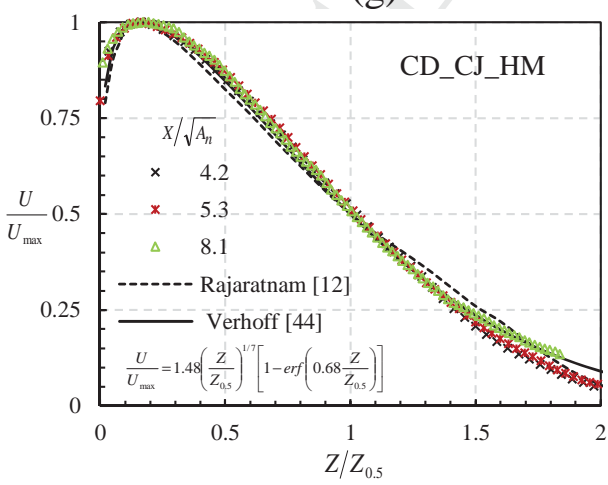

(b)

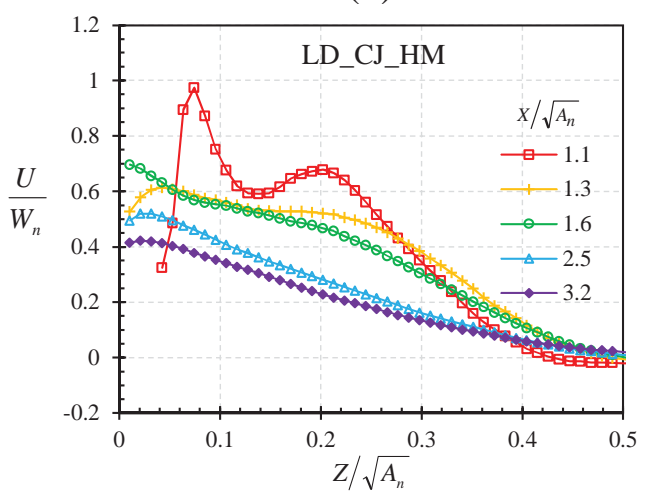

(d)

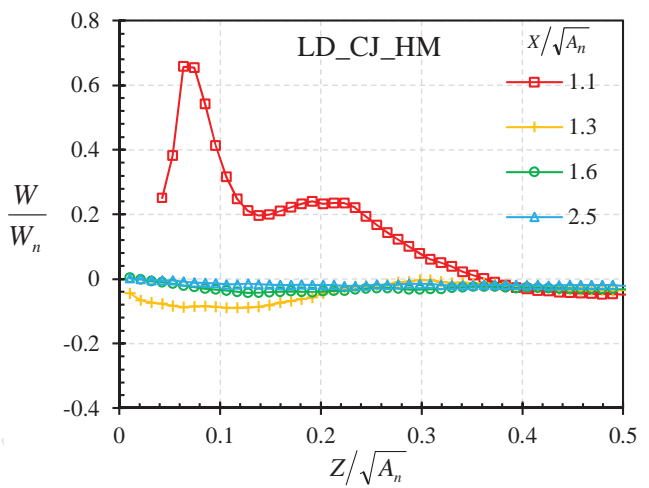

(f)

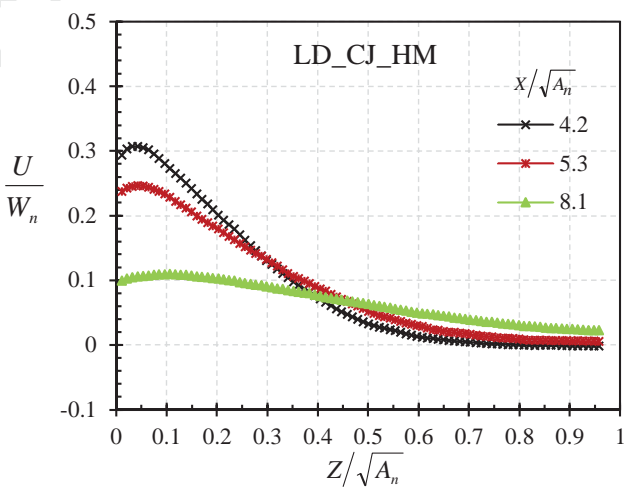

(h)

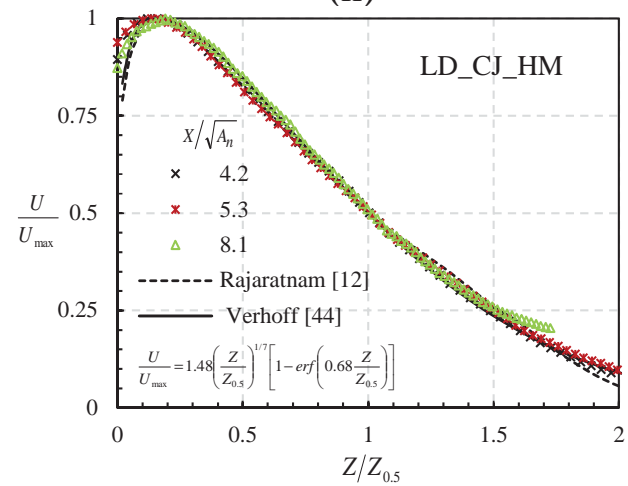

Fig. 7 (a-f) Radial $(U)$ and vertical $(W)$ velocity profiles of the ceiling jet at different normalized distances from the difuser outlet. (g,h) Comparison of $U / U_{\max }$ profiles with experimental data of the litterature [12] and with the velocity distribution equation of Verhoff given for a radial academic jet [44] 
(a)

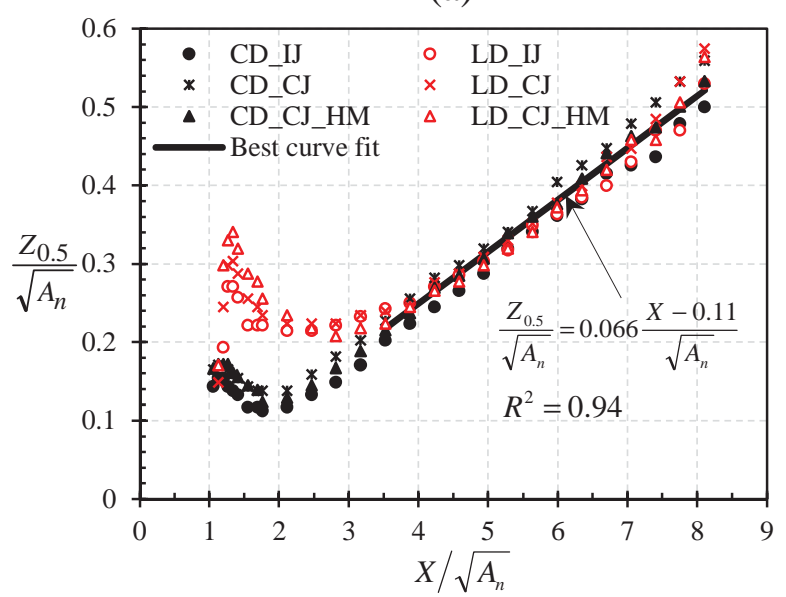

(c)

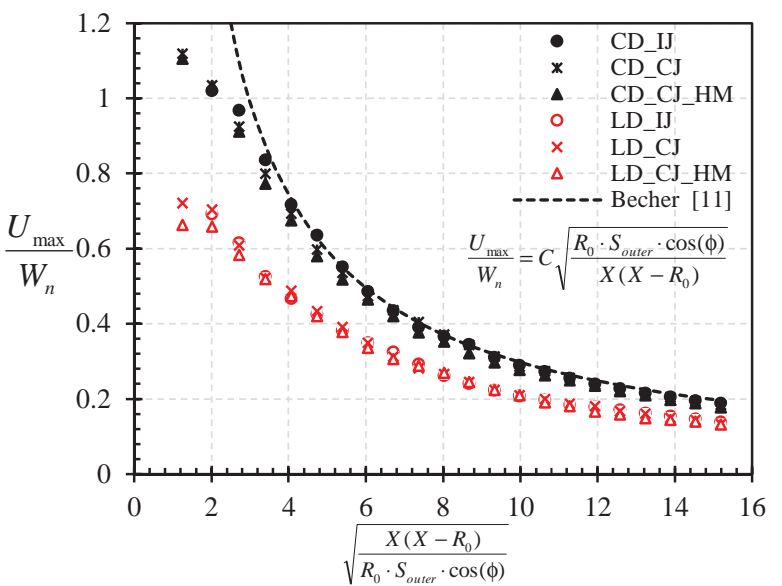

(b)

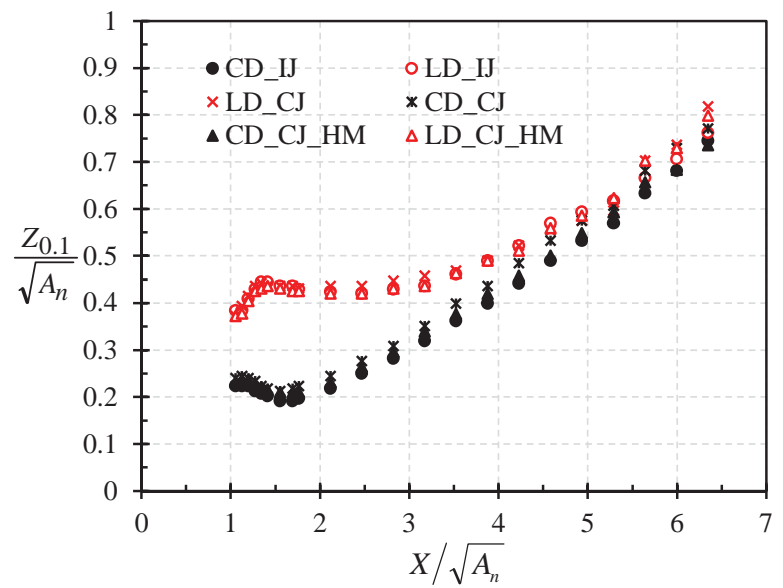

(d)

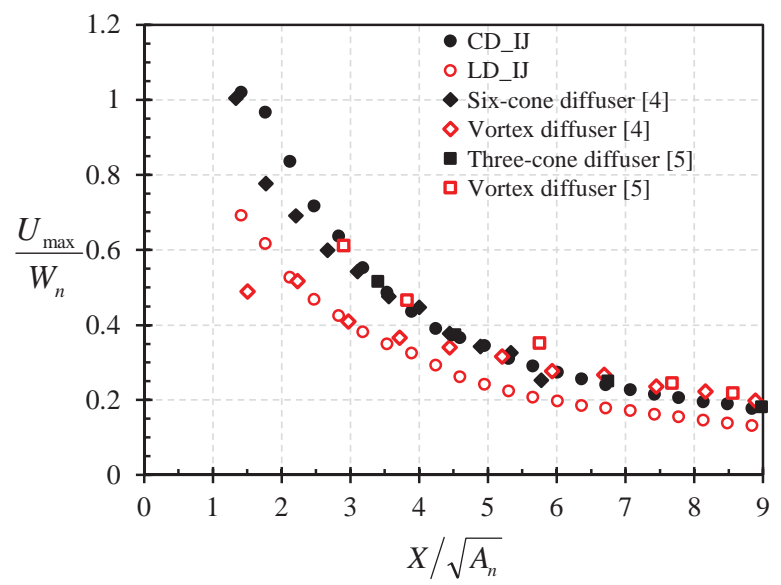

Fig. 8 (a, b) Jet widths $Z_{0.5}$ and $Z_{0.1}$; (c, d) Maximum jet velocity decay in two different representations: (c) comparison with Becher equation [11] with $C=3.1$, (d) comparison with available literature data $[4,5]$ 


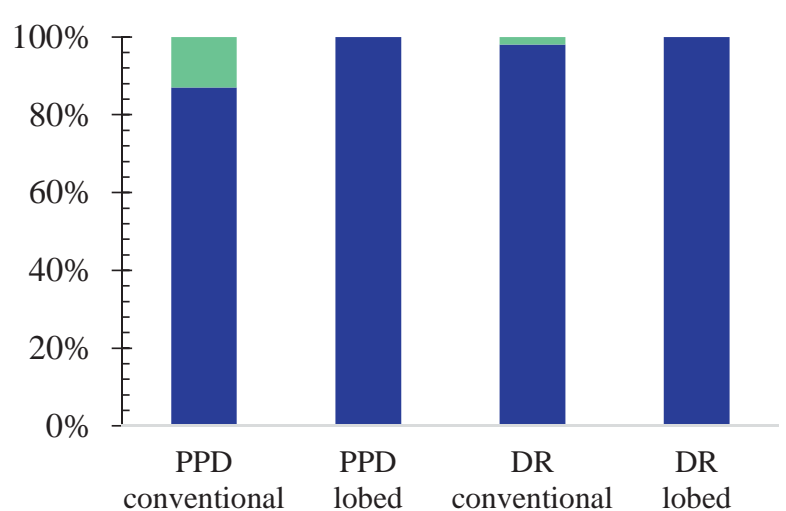

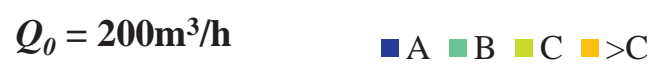

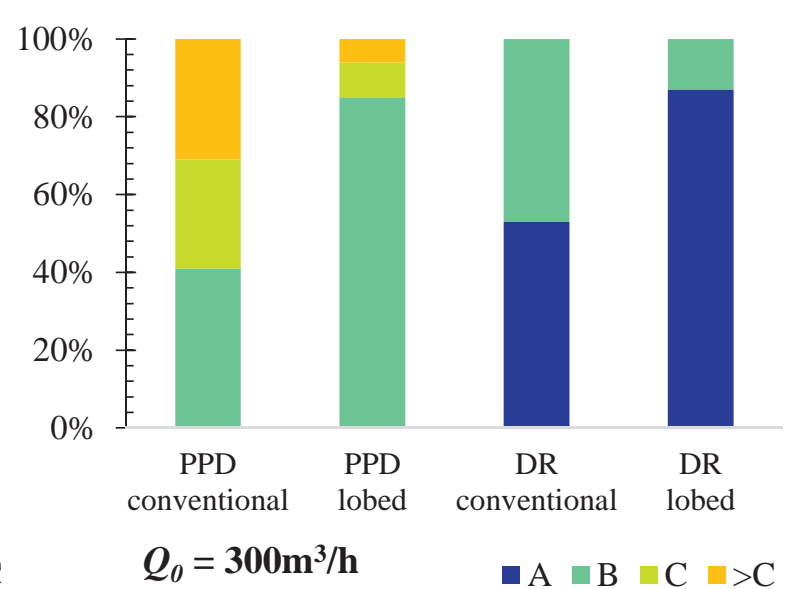

Fig. 9 Statistic distribution of $P P D$ and DR indices for the two diffusers, in accordance of ISO 7730 standard 

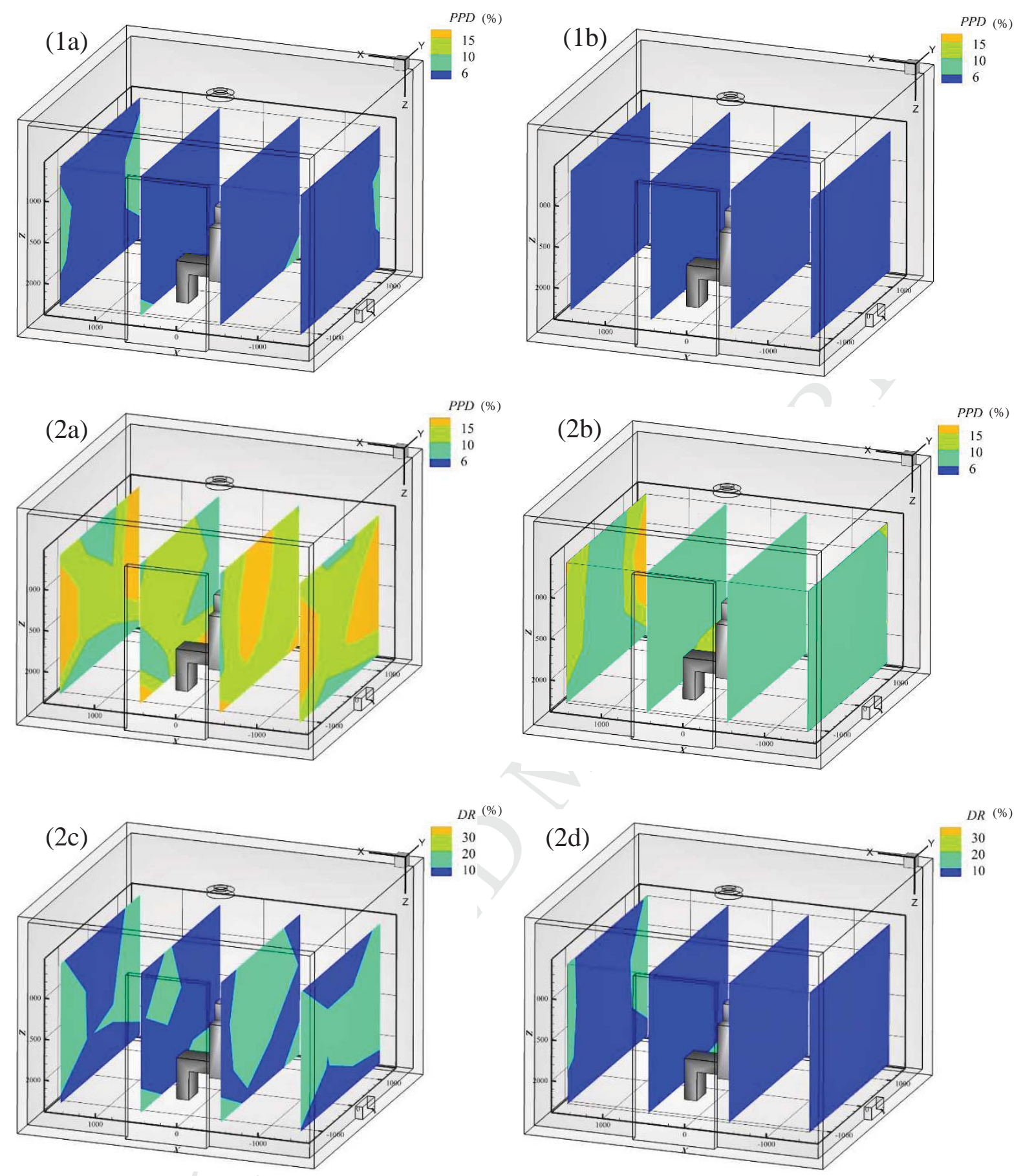

Fig. 10 Distributions of PPD (a,b) and DR (c,d) in CD_CJ_HM (left) and LD_CJ_HM (right) cases: (1) $Q_{0}=200 \mathrm{~m}^{3} / \mathrm{h}$, (2) $Q_{0}=300 \mathrm{~m}^{3} / \mathrm{h}$ 

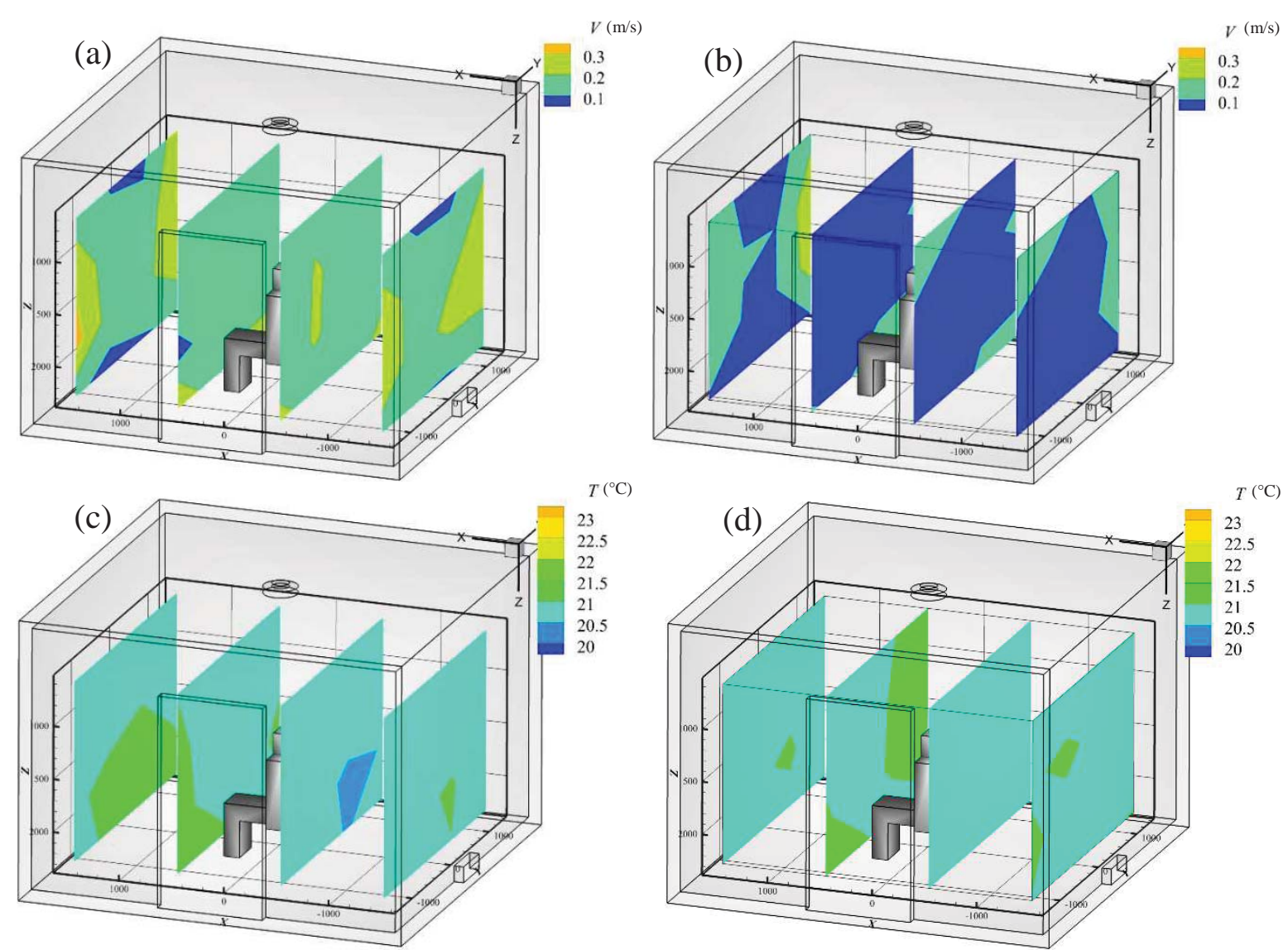

(e)

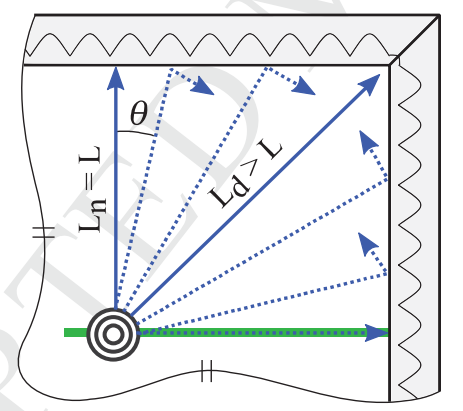

Fig. 11 Distributions of velocity magnitude (a, b), and air temperature (c, d) for $Q_{0}=300$ $\mathrm{m}^{3} / \mathrm{h}$, in CD_CJ_HM (left) and LD_CJ_HM (right). (e) Sketch of ceiling radial jet impingement on the wall and of confluence of downhill flows towards a corner - the green line symbolizes the laser plane in LS-PIV measurements 
(a)

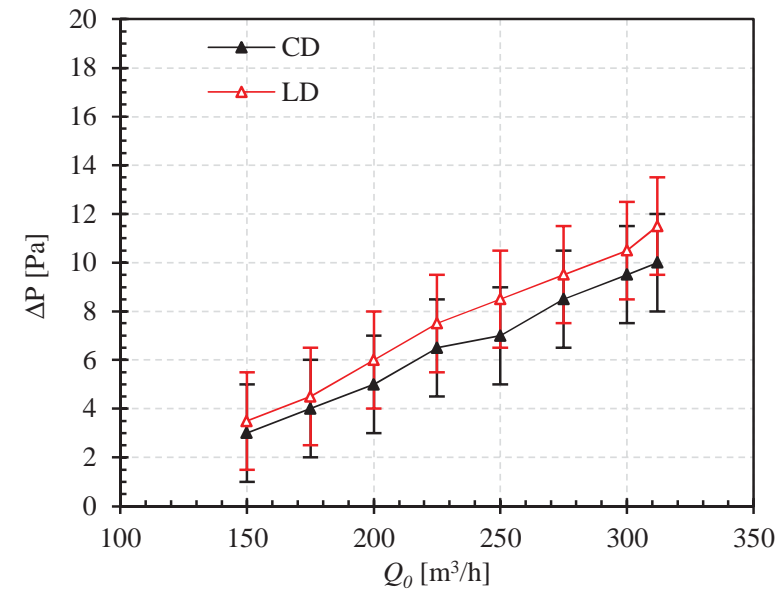

(b)

(c)

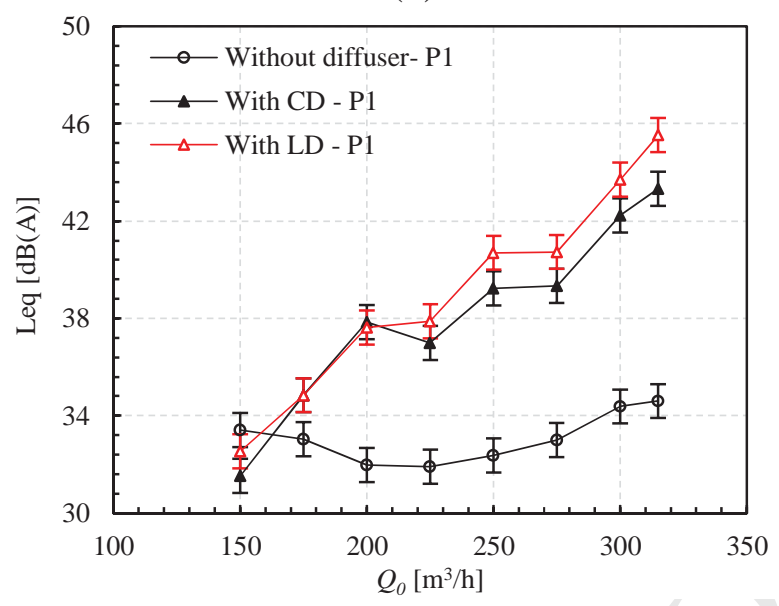

(d)

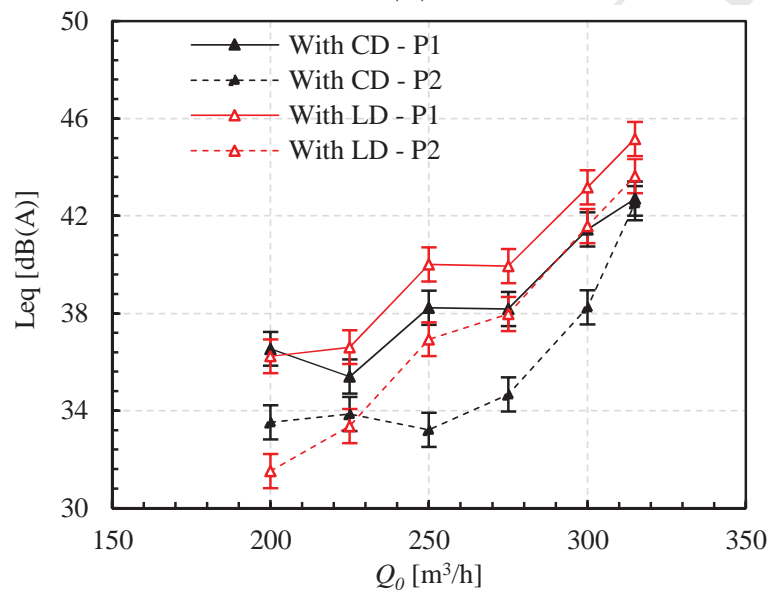

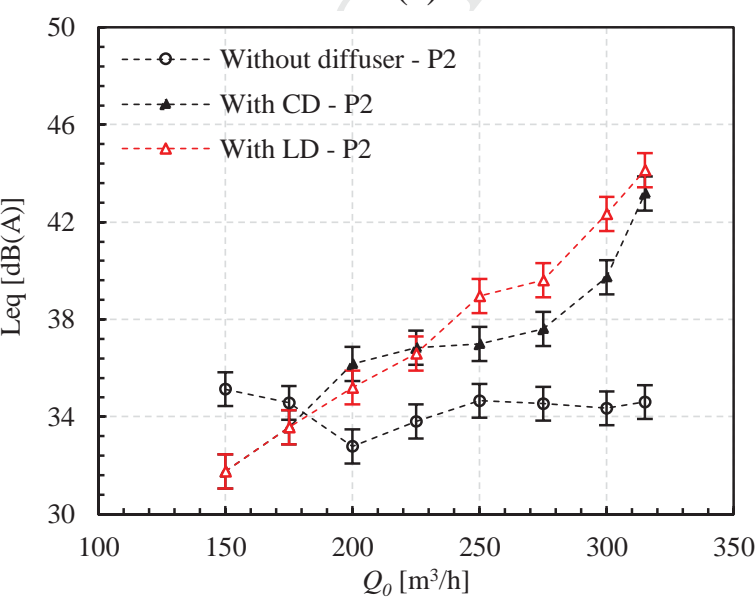

(e)

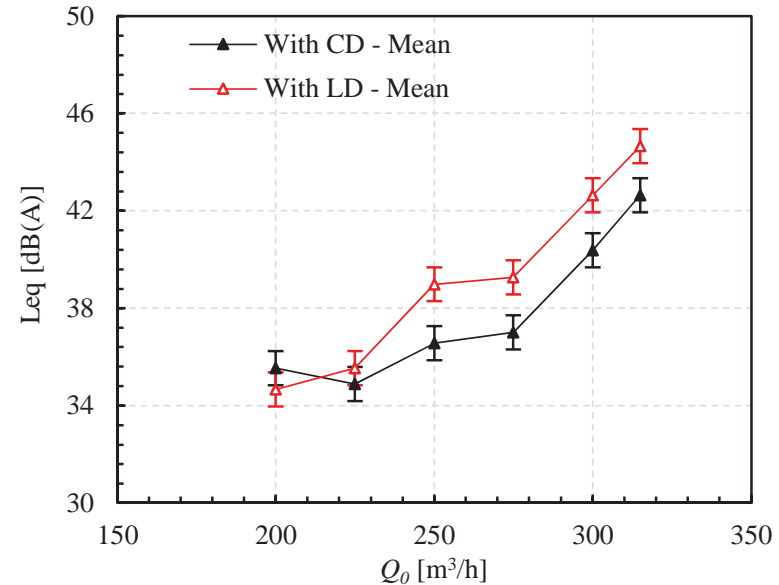

Fig.12 (a) Total pressure loss of the two diffusers with error bar of \pm 2 Pa; (b-e) Global sound pressure level (error bar of $\pm 0.7 \mathrm{~dB}$ ) of the diffusers and the ventilation equipment measured at the locations P1 and P2 of Fig. 1, a 


\section{$\underline{\text { Research Highlights: }}$}

$>$ Flow pattern and thermal comfort are analyzed in a room under mixing ventilation $>\mathrm{A}$ radial cold jet is generated by a conventional ceiling multi-cone diffuser $>$ The effect of inserted lobes into the conventional diffuser is analyzed > Significant improvement of thermal comfort is achieved due to jet induction increase $>$ It was not observed a significant increase in pressure drop and noise 